\title{
THE WEYL PRINCIPLE ON THE FINSLER FRONTIER
}

\author{
DMITRY FAIFMAN AND THOMAS WANNERER
}

\begin{abstract}
Any Riemannian manifold has a canonical collection of valuations (finitely additive measures) attached to it, known as the intrinsic volumes or Lipschitz-Killing valuations. They date back to the remarkable discovery of $\mathrm{H}$. Weyl that the coefficients of the tube volume polynomial are intrinsic invariants of the metric. As a consequence, the intrinsic volumes behave naturally under isometric immersions. This phenomenon, subsequently observed in a number of different geometric settings, is commonly referred to as the Weyl principle. In general normed spaces, the Holmes-Thompson intrinsic volumes naturally extend the Euclidean intrinsic volumes. The purpose of this note is to investigate the applicability of the Weyl principle to Finsler manifolds. We show that while in general the Weyl principle fails, a weak form of the principle unexpectedly persists in certain settings.
\end{abstract}

\section{INTRODUCTION}

The relation between the intrinsic and extrinsic geometric properties of submanifolds of a finite-dimensional normed space is not well understood, see, e.g., 8, 17, 27]. It is not even clear which intrinsic and extrinsic quantities can be related to each other: D. Burago and S. Ivanov [17] proved the striking result that while geodesics on Finslerian saddle surfaces exhibit the same global behaviour as in the Riemannian case, there seem to be no special local properties of saddle surfaces that imply these global results, as every abstract Finsler surface can be locally embedded as a saddle surface in some 4-dimensional normed space.

In convex geometry, the central global invariants of a convex body $K$ in Euclidean space $\mathbb{R}^{n}$ are its intrinsic volumes. They arise as the (suitably normalized) coefficients in Steiner's formula for the volume of an $\varepsilon$-neighborhood of $K$. The intrinsic volumes of $K \subset \mathbb{R}^{m} \subset \mathbb{R}^{n}$ do not depend on the ambient space, i.e. are the same whether computed in $\mathbb{R}^{m}$ or in $\mathbb{R}^{n}$. This fact was generalized to compact smooth submanifolds $M$ of $\mathbb{R}^{n}$ by H. Weyl [38], who expressed the intrinsic volumes in terms of polynomial invariants of the Riemannian curvature tensor of $M$. As a function on $\mathcal{K}\left(\mathbb{R}^{n}\right)$, the space of convex bodies in $\mathbb{R}^{n}$, the $k^{\text {th }}$ intrinsic volume $\mu_{k}$ possesses a strikingly simple characterization: $\mu_{k}$ is the unique even, $k$-homogeneous, translation-invariant, continuous valuation, i.e., satisfies

$$
\mu_{k}(K \cap L)=\mu_{k}(K)+\mu_{k}(L)-\mu_{k}(K \cap L)
$$

Date: December 20, 2019.

DF partially supported by NSERC Discovery Grant.

TW supported by DFG grant WA 3510/1-1. 
whenever $K \cup L$ is convex, with the property that

$$
\left.\mu_{k}\right|_{E}=\operatorname{vol}_{E}
$$

for every $k$-dimensional linear subspace $E \subset \mathbb{R}^{n}$, where $\operatorname{vol}_{E}$ denotes the usual Lebesgue measure on $E$. This follows at once from the Klain embedding theorem 1, 28,

The Holmes-Thompson intrinsic volumes naturally extend the notion of the Euclidean intrinsic volumes to general smooth Minkowski spaces, i.e. finite-dimensional normed spaces with a smooth unit ball $B_{F}$ of positive gaussian curvature. There are several natural ways to normalize the Lebesgue measure on a normed space $V$, see e.g. [11, or the book by A.C. Thompson [37]. From the perspective of integral geometry, as well as symplectic geometry, the natural definition is the Holmes-Thompson volume, given by setting

$$
\operatorname{vol}^{H T}\left(B_{F}\right)=\frac{1}{\omega_{n}} \operatorname{vol}_{2 n}\left(B_{F} \times B_{F}^{\circ}\right)
$$

where $B_{F}^{\circ} \subset V^{*}$ is the dual convex body, $\omega_{n}$ the volume of the Euclidean unit ball, and $\operatorname{vol}_{2 n}$ the Liouville volume for the standard symplectic form on $V \times V^{*}$. The normalizing factor $1 / \omega_{n}$ is chosen so that $\mathrm{vol}^{H T}$ extends the standard Euclidean definition of volume. If $E \subset V$ is a linear subspace, it inherits a norm and therefore the corresponding Holmes-Thompson Lebesgue measure $\operatorname{vol}_{E}^{H T}$. More generally, given a $k$-dimensional submanifold $X \subset V$, we get the Holmes-Thompson volume measure $\operatorname{vol}_{X}^{H T}$ on $X$, since an absolutely continuous measure on a manifold is canonically identified with a continuous choice of a Lebesgue measure on its tangent spaces. It was shown by J.C. Álvarez Paiva and E. Fernandes [10] and A. Bernig [12] that the $k$-dimensional Holmes-Thompson volume can be extended to a valuation on $\mathcal{K}(V)$. Namely, one can define the $k^{\text {th }}$ Holmes-Thompson intrinsic volume as the unique even, $k$-homogeneous, translation-invariant, continuous valuation satisfying

$$
\left.\mu_{k}^{F}\right|_{E}=\operatorname{vol}_{E}^{H T}
$$

for every $k$-dimensional linear subspace $E \subset V$. The uniqueness of the extension of $\operatorname{vol}_{k}^{H T}$ follows as in the Euclidean case from the Klain embedding theorem. As a consequence of this uniqueness, the Holmes-Thompson intrinsic volumes are intrinsic in the following sense: If $K \subset U \subset V$ is a convex body contained in a linear subspace $U \subset V$ with the induced norm, then the value of $k$ th Holmes-Thompson intrinsic volume on $K$ is the same whether computed in $(V, F)$ or $\left(U,\left.F\right|_{U}\right)$. We will refer to this property as the linear Weyl principle. As their Euclidean counterparts, the Holmes-Thompson intrinsic volumes may be evaluated on much more general objects than convex bodies, in particular on compact smooth submanifolds with boundary (see Section 2).

A guiding idea in valuation theory, usually referred to as the Weyl principle, has recently emerged: when restricted to a subspace $X$, valuations on an ambient space $M$ may often be reconstructed from the basic geometry of $X$ induced by the immersion into $M$. The prototypical application is Weyl's theorem described above, but more recently several other instances have surfaced: immersions of contact and 
dual Heisenberg manifolds and restriction of their canonical valuations [18]; restriction of the invariant valuations on complex space forms to totally real submanifolds [15. Lemma 4.4]; and immersions of pseudo-Riemannian manifolds and the restriction of their canonical valuations [14].

Approaching the question of intrinsic and extrinsic geometric properties of submanifolds of a finite-dimensional normed space from an integral geometric perspective, J.H.G. Fu put forward the conjecture [6, p. 107] that the Weyl principle also applies to the Holmes-Thompson intrinsic volumes. More precisely, let $V$ be an $n$-dimensional real vector space, and let $F: V \rightarrow \mathbb{R}$ be a smooth norm on $V$. The pullback of a valuation $\mu$ on $V$ under a smooth embedding $e: M \rightarrow V$ is defined by

$$
e^{*} \mu(A)=\mu(e(A)),
$$

where $A \subset M$ is a compact submanifold with corners (see Section 2). This definition extends naturally to smooth immersions. The conjecture of J.H.G. Fu can now be stated as follows.

Conjecture 1.1 ([6, p. 107]). Let $e_{j}: M \rightarrow V_{j}, j=1,2$ be smooth immersions of a smooth manifold $M$ into the normed spaces $\left(V_{j}, F_{j}\right)$. If $e_{1}^{*} F_{1}=e_{2}^{*} F_{2}$, then

$$
e_{1}^{*} \mu_{k}^{F_{1}}=e_{2}^{*} \mu_{k}^{F_{2}} \text {. }
$$

When $\operatorname{dim} M \leq 2$, the conjecture is easily seen to hold (Theorem 2.6). The first unknown case is $\operatorname{dim} M=3, \operatorname{dim} V=4$.

D. Burago and S. Ivanov proved [16] a Burstin-Janet-Cartan theorem in the Finsler setting, namely that every Finsler manifold can be locally isometrically embedded in a normed space. Thus a positive answer to the conjecture would allow to define the Holmes-Thompson valuations on arbitrary Finsler manifolds.

1.1. Main results. In loose terms, the Finslerian setting allows an extra freedom compared with the Riemannian setting. Namely, we may vary not only the immersion, but also the ambient normed space itself, so long as the restricted norm is unchanged.

We refute Conjecture 1.1 in general, while simultaneously proving that several weakened versions of the Weyl principle persist in the Finsler setting. We find that in some settings, keeping the immersion fixed, the restricted Holmes-Thompson intrinsic volumes are independent of the ambient norm. To give precise statements, let us introduce some terminology. All norms are assumed to have a smooth and positively curved unit ball.

Definition 1.2. Let $M \subset V$ be an $m$-dimensional manifold without boundary, immersed in an $n$-dimensional linear space $V$. We say that $M$ satisfies the weak Weyl principle $(W W P)$ at $p \in M$ if whenever $F, F^{\prime}$ are two norms on $V$ that coincide on $\left\{T_{x} M: x \in U\right\}$ for some neighborhood $U$ of $p$, it also holds that $\left.\mu_{k}^{F}\right|_{U}=\left.\mu_{k}^{F^{\prime}}\right|_{U}$, for all $k \geq 1$. We say that $M \subset V$ satisfies $W W P$ if it holds for all $p \in M$.

Our first main result states that the weak Weyl principle holds unconditionally for small codimension, and furthermore holds generically when the codimension is not too large, as follows. 
Theorem A. Fix a manifold $M$, assume $\operatorname{dim} M=m$ and $\operatorname{dim} V=n$. If $n \leq m+2$ then WWP holds for any immersion e : $M \rightarrow V$. If $m+3 \leq n \leq 2 m$, then $W W P$ holds for a dense residual set of immersions $M \rightarrow V$ in the Whitney topology.

Here by a residual set we mean the countable intersection of open dense sets.

Our second main theorem shows that WWP fails for generic submanifolds of large codimension. We henceforth make use of an auxiliary Euclidean structure on $V=\mathbb{R}^{n}$, and write $S(V)=S^{n-1}$ for the unit sphere. We get an induced Riemannian structure on $M$, and define the tautological map $\theta: S M \rightarrow S^{n-1}$ by $\theta(x, v)=v$, where $S M$ denotes the unit tangent bundle.

Definition 1.3. A manifold $M \subset V$ is directionally regular at $(p, u) \in S M$ if the differential $d_{(p, u)} \theta: T_{(p, u)} S M \rightarrow T_{u} S^{n-1}$ has full rank, namely $\min (2 m-1, n-1)$.

Recall that the $2^{\text {nd }}$ osculating space $\mathcal{O}_{p}^{2} M \subset T_{p} V$ at $p \in M$ is spanned by the velocity and acceleration vectors of all curves at $p$. The Euclidean orthogonal complement of $T_{p} M$ in $\mathcal{O}_{p}^{2} M$ is the $1^{\text {st }}$ normal space $\mathcal{N}_{p}^{1} M$ (see, e.g., [35]).

Theorem B. Assume $m \geq 3$, and $M^{m} \subset V$ is directionally regular at $(p, u) \in S M$. Then $W W P$ for $M$ fails at $p$ if $\operatorname{dim} \mathcal{O}_{p}^{2} M>2 m$.

Since the $2^{\text {nd }}$ osculating space is generically of dimension $m+\min \left(\frac{m(m+1)}{2}\right.$, codim $\left.M\right)$, WWP generically fails when $\operatorname{codim} M>m \geq 3$. In particular, Conjecture 1.1 fails for $\operatorname{dim} M \geq 3$.

Finally, we observe that a variant of the weak Weyl principle holds in general if we restrict not only the norm but also its derivatives, as follows.

Theorem C. $\left.\mu_{k}^{F}\right|_{M}$ is determined by the values $\frac{\partial^{j} F}{\partial v^{j}}(u)$, for all $(p, u) \in S M$ and $v \in \mathcal{N}_{p}^{1} M \subset T_{u} S^{n-1}$, for all $j \leq m-1$.

According to [25], every $m$-dimensional Finsler manifold can be locally isometrically embedded in a $2 m$-dimensional normed space, see also 33 and the references therein. In this situation, WWP holds by Theorem A for a generic submanifold, but fails in general except in codimensions 1 and 2. Moreover, we give an explicit example of a submanifold $M^{3} \subset \mathbb{R}^{6}$ for which WWP fails (Example 5.6), which is the lowest dimensional setting when this can happen. Thus the genericity assumption in Theorem $\mathrm{A}$ is essential, and one cannot define Holmes-Thompson intrinsic volumes on general Finsler manifolds.

Let us comment on the choice of the Holmes-Thompson definition of volume for the generalization of the Euclidean intrinsic volumes to smooth Minkowski spaces. Given a definition of volume $\mathcal{V}$ for normed spaces, one may ask if $k$-homogeneous valuations can be functorially assigned to a normed space $(V, F)$ for each $0 \leq k \leq$ $\operatorname{dim} V$, such that $\left.\mu_{k}^{\mathcal{V}}\right|_{E}=\mathcal{V}_{\left(E,\left.F\right|_{E}\right)}$ for each $k$-dimensional linear subspace $E \subset V$. By the Klain embedding theorem, $\mu_{k}^{\mathcal{V}}$ would satisfy the linear Weyl principle. According to J.C. Álvarez Paiva [8], the three most commonly used definitions of volume in Finsler Geometry are: Busemann, Holmes-Thompson, and Benson (Gromov mass*). To date, only the Holmes-Thompson definition has been shown to be extendable as a family of valuations. Moreover, the Holmes-Thompson intrinsic volumes form an 
algebra under the Alesker product [12], and no other definition of volume would have this property. This follows at once by noting that all definitions of volume coincide for 1-dimensional spaces, and by the Klain embedding theorem, the corresponding 1-homogeneous valuation must coincide for all definitions of volume. This makes the Holmes-Thompson definition the most natural from the perspective of integral geometry (see also [9, 29, 32] for further evidence).

It is worth noting that the Weyl principle will be seen to fail already for the 1homogeneous valuations. Thus the Weyl principle would fail in the Finsler setting for any definition of volume which can be extended to a family of valuations.

1.2. Plan of the paper. In Section 2, we recall the notions of valuation theory that we need and carry out the simple reduction to the 1-homegeneous case. In Section 3, we compute the defining current of the first Holmes-Thompson intrinsic volume. Then in Section 4, we compute explicitly the restriction to a submanifold, which is given by certain fiber integrals. We then utilize classical harmonic analysis on the sphere to simplify those integrals. Finally in Section 5 we utilize the previous computation to prove the main theorems, and construct some examples demonstrating the sharpness of our results. Section 6 is devoted to open problems and conjectures.

\section{BACKGROUND AND PREPARATION}

2.1. Some integral transforms. We write $C_{\epsilon}^{N}\left(S^{n-1}\right)$ for the space of $C^{N}$-smooth functions on the sphere of parity $\epsilon= \pm$ with respect to the antipodal map.

We will use the following $\mathrm{SO}(n)$-equivariant integral operators:

(1) The cosine transform $\mathcal{C}: C^{\infty}\left(S^{n-1}\right) \rightarrow C^{\infty}\left(S^{n-1}\right)$ given by

$$
\mathcal{C}(f)(v)=\int_{S^{n-1}}|\langle u, v\rangle| f(u) d u
$$

(2) The hemispherical transform $H: C^{\infty}\left(S^{n-1}\right) \rightarrow C^{\infty}\left(S^{n-1}\right)$ given by

$$
H(f)(v)=\int_{u \in S^{n-1}:\langle u, v\rangle \geq 0} f(u) d u
$$

When ambiguity can arise, we write $\mathcal{C}_{n}$ instead of $\mathcal{C}$. It is clear that $\mathcal{C}$ vanishes on odd functions, and $\mathcal{C}$ is well-known to be invertible on the even functions, with a continuous inverse $\mathcal{C}^{-1}: C_{+}^{\infty}\left(S^{n-1}\right) \rightarrow C_{+}^{\infty}\left(S^{n-1}\right)$, see, e.g., [24]. In contrast, $H$ has rank one on the even functions, and is continuously invertible on the odd functions 30.

The $s$ th Sobolev norm on $S^{m-1}$ is given by

$$
\|f\|_{H^{s}}=\left(\sum_{n \geq 0}(1+n)^{2 s}\left\|f_{n}\right\|_{L^{2}}^{2}\right)^{1 / 2},
$$

where $f=\sum_{n} f_{n}$ is the decomposition into spherical harmonics, and the Sobolev space $H^{s}\left(S^{m-1}\right)$ is the completion of $C^{\infty}\left(S^{m-1}\right)$ with respect to this norm (see, e.g., 
[22]). The $C^{k}$-norm on $S^{m-1}$ is defined inductively by

$$
\|f\|_{C^{k}}=\|f\|_{C^{0}}+\sup _{i}\left\|X_{i} f\right\|_{C^{k-1}},
$$

where $\left(X_{i}\right)$ is a basis of the Lie algebra $\mathfrak{s o}(n)$ and $X f(u)=\left.\frac{d}{d t}\right|_{t=0} f\left(e^{t X} u\right)$ for each $X \in \mathfrak{s o}(n)$. By [24, Eq. 3.6.4], there is a continuous embedding

$$
C^{2 k}\left(S^{m-1}\right) \subset H^{s}\left(S^{m-1}\right) \quad \text { for } 2 k>s+\frac{n}{2} ;
$$

By the Sobolev embedding theorem,

$$
H^{s}\left(S^{m-1}\right) \subset C^{k}\left(S^{n-1}\right) \quad \text { for } s>k+\frac{n-1}{2} .
$$

In particular, $\bigcap_{s>0} H^{s}\left(S^{m-1}\right)=C^{\infty}\left(S^{m-1}\right)$.

2.2. Valuations. As a general reference for valuation theory we recommend the books by R. Schneider [31, Chapter 6] and S. Alesker [7].

We write $\operatorname{Dens}(V)$ for the one-dimensional space of Lebesgue measures on the finite-dimensional real vector space $V$. The even $k$-homogeneous valuations on $V$ are denoted $\mathrm{Val}_{k}^{+}(V)$. The Klain map $\mathrm{Kl}: \mathrm{Val}_{k}^{+}(V) \rightarrow \Gamma\left(\mathrm{Gr}_{k}(V)\right.$, Dens $\left.(E)\right)$ is given by $\mathrm{Kl}_{\phi}=\left.\phi\right|_{E}$. The latter is a Lebesgue measure on $E$ by Hadwiger's theorem. By a theorem of Klain, $\mathrm{Kl}$ is injective.

Put $\mathbb{P}_{M}=\mathbb{P}_{+}\left(T^{*} M\right)$ for the co-sphere bundle of $M$, which is the oriented projectivization of the cotangent bundle. It is equipped with a natural contact structure, and we call a form $\omega \in \Omega\left(\mathbb{P}_{M}\right)$ vertical if its restriction to any contact hyperplane vanishes. Given a Riemannian structure on $M$, there is a natural choice of contact form $\alpha$ given by $\alpha_{x, \xi}=\langle\xi, d \pi(\bullet)\rangle$, where $\pi: \mathbb{P}_{M} \rightarrow M$ is the natural projection, under the identification $\mathbb{P}_{M}=S M$.

Let us recall more precisely the notion of valuations on manifolds. For simplicity, we assume $M$ is oriented. The family of test bodies $\mathcal{P}(M)$ can be chosen in several ways; we take them to be the compact submanifolds with corners. For $X \in \mathcal{P}(M)$, its conormal cycle is nc $(X) \subset \mathbb{P}_{M}$. It generalizes both the normal cycle of a convex set in a linear space and the conormal bundle of a submanifold, see, e.g. [19 21

Definition 2.1. The space of smooth valuations $\mathcal{V}^{\infty}(M)$ on an oriented manifold $M^{m}$ consists of functions $\phi: \mathcal{P}(M) \rightarrow \mathbb{R}$ of the form

$$
\phi(X)=\int_{X} \mu+\int_{\mathrm{nc}(X)} \omega
$$

where $\mu \in \Omega^{m}(M)$ and $\omega \in \Omega^{m-1}\left(\mathbb{P}_{M}\right)$.

It was established in 13 that a smooth valuation $\phi \in \mathcal{V}^{\infty}(M)$ is uniquely determined by its defining currents $(C, T) \in C^{\infty}(M) \times \Omega^{m}\left(\mathbb{P}_{M}\right)$, which can be any pair of forms subject to the conditions

(1) $T$ is closed and vertical.

(2) $\pi_{*} T=d C$, where $\pi: \mathbb{P}_{M} \rightarrow M$ is the projection.

If $\phi \in \mathcal{V}^{\infty}(M)$ is represented by $(\omega, \mu)$ then $T=a^{*}\left(D \omega+\pi^{*} \mu\right), C=\pi_{*} \omega$ where $a$ is the fiberwise antipodal map, and $D$ the Rumin differential operator. We write $\phi=[[\mu, \omega]]=[(C, T)], T=T(\phi), C=C(\phi)$. 
Every immersion $e: M \leftrightarrow N$ of smooth manifolds induces a pullback map $e^{*}$ : $\mathcal{V}^{\infty}(N) \rightarrow \mathcal{V}^{\infty}(M)$, which for embeddings is given by

$$
e^{*} \phi(X)=\phi(e(X)), \quad X \in \mathcal{P}(M) .
$$

On the level of differential forms and in the special case $e: M \rightarrow V$ it can be described as follows (see [2]). Let $N^{*} M$ denote the conormal bundle and consider the following natural maps: the projection $q: \mathbb{P}_{+}\left(N^{*} M\right) \rightarrow M$; the fiberwise inclusion $\theta: \mathbb{P}_{+}\left(N^{*} M\right) \leftrightarrow \mathbb{P}_{V}$; the map $\beta: \mathbb{P}_{V} \times_{V} M \rightarrow \mathbb{P}_{M}$, extending the projection $\mathbb{P}_{V} \times_{V} M \backslash N^{*} M \rightarrow \mathbb{P}_{M}$ to $\mathbb{P}_{V} \times_{V} M$, which is the oriented blow-up along $N^{*} M$; and $\bar{\alpha}: \mathbb{P}_{V} \times_{V} M \rightarrow \mathbb{P}_{V}$, the composition of the blow-up map with the natural map $\mathbb{P}_{V} \times_{V} M \rightarrow \mathbb{P}_{V}$. If $\phi \in \mathcal{V}^{\infty}(V)$ is represented by $(\mu, \omega) \in \Omega^{n}(M) \times \Omega^{m-1}\left(\mathbb{P}_{M}\right)$, then $e^{*} \phi=\left[\left[\mu^{\prime}, \omega^{\prime}\right]\right]$ where

$$
\omega^{\prime}=\beta_{*} \bar{\alpha}^{*} \omega, \quad \mu^{\prime}=q_{*} \theta^{*} \omega
$$

Alternatively, if $\phi=[(C, T)]$, then $e^{*} \phi=\left[\left(C^{\prime}, T^{\prime}\right)\right]$ where

$$
T^{\prime}=\beta_{*} \bar{\alpha}^{*} T, \quad C^{\prime}=e^{*} C .
$$

2.3. The trivial cases. Here we verify Conjecture 1.1 for $\operatorname{dim} M \leq 2$ and show that in general it is enough to prove it for the first intrinsic volume. In the following, $V$ is a finite-dimensional real vector space.

Lemma 2.2. Let $\varphi \in \operatorname{Val}_{k}^{+}(V)$ be a smooth valuation. If $X \in \mathcal{P}(V)$ is a compact $k$-dimensional submanifold with corners, then

$$
\varphi(X)=\int_{X} \mathrm{Kl}_{\varphi}\left(T_{x} M\right)
$$

Proof. Similar to the proof of Lemma 3.7(ii) in [34].

The following is a direct consequence of the above lemma and the definitions.

Corollary 2.3. Let $V$ be a normed space. If $X \in \mathcal{P}(V)$ is a compact $k$-dimensional manifold with corners, then $\mu_{k}^{F}(X)$ equals the Holmes-Thompson volume of $\left(X,\left.F\right|_{X}\right)$. If $M \subset V$ is a k-dimensional immersed submanifold, then $\left.\mu_{k}^{F}\right|_{M}$ is the HolmesThompson volume measure of $\left(M,\left.F\right|_{M}\right)$.

Lemma 2.4. Consider a smooth valuation $\varphi \in \mathrm{Val}_{k}^{+}(V)$. If $X \subset V$ is a compact $(k+1)$-dimensional submanifold with boundary, then

$$
\varphi(X)=\frac{1}{2} \varphi(\partial X)
$$

Proof. Note that $\phi$ has Euler-Verdier eigenvalue equal to $(-1)^{k}$. Now apply 3 , Lemma 4.1.1].

Corollary 2.5. Let $(V, F)$ be a normed space and let $M \subset V$ be an immersed smooth submanifold of dimension $k+1$. Then the restriction of $\mu_{k}^{F}$ to $M$ is intrinsically defined. For every compact submanifold with corners $X \subset M, \mu_{k}^{F}(X)=$ $\frac{1}{2} \operatorname{vol}_{k}^{H T}\left(\partial X,\left.F\right|_{\partial X}\right)$. 
Proof. For $X$ a submanifold with boundary, this follows from Lemma 2.4 and Corollary [2.3. As the proof of [13, Theorem 1] shows that a smooth valuation is determined by its values on smooth submanifolds with boundary, we conclude that $\left.\mu_{k}^{F}\right|_{M}$ is intrinsically defined. For the last statement, we may use continuity and approximate a manifold with corners by a sequence of manifolds with boundary.

In particular, the Weyl principle holds for Finsler surfaces.

Theorem 2.6. Conjecture 1.1 holds for $\operatorname{dim} M \leq 2$.

Proof. This is an immediate consequence of Corollaries 2.3 and 2.5 .

Finally, we observe that it suffices to prove the Weyl principle for the first intrinsic volume.

Proposition 2.7. Let $M \subset V$ be a submanifold. If $\left.\mu_{1}^{F}\right|_{M}$ is intrinsically defined, then so are all $\left.\mu_{k}^{F}\right|_{M}$ for all $k \geq 2$.

Proof. This follows at once by applying the Alesker product. It holds that $\mu_{k}^{F}=$ $c_{k}\left(\mu_{1}^{F}\right)^{k}$ for certain universal coefficients $c_{k}$ [12], and it remains to recall that the Alesker product commutes with restrictions.

\section{The DEFINING CURRENT OF THE FIRST INTRINSIC VOlume}

For the remainder of the paper, we fix an auxiliary Euclidean structure on $V=\mathbb{R}^{n}$, and let $S^{n-1}$ be the corresponding unit sphere.

Lemma 3.1. It holds that

$$
T\left(\mu_{1}^{F}\right)=c_{n} \mathcal{C}^{-1}(F) \alpha \wedge \operatorname{vol}_{S^{n-1}}, \quad C\left(\mu_{1}^{F}\right)=0,
$$

where $c_{n}$ is a constant depending only on $n$, and $\alpha$ is the contact form.

Proof. Write the cosine transform in a GL( $V$ )-equivariant form (see [5]):

$$
\mathcal{C}: C^{\infty}\left(\operatorname{Gr}_{n-1}(V), \operatorname{Dens}(V / E) \otimes|\omega|\right) \rightarrow C^{\infty}\left(\operatorname{Gr}_{1}(V), \operatorname{Dens}(E)\right),
$$

where $|\omega|=\operatorname{Dens}\left(T_{E} \mathrm{Gr}_{n-1}(V)\right)$ is the line bundle of smooth densities on $\operatorname{Gr}_{n-1}(V)$. As the Klain section of $\mu_{1}^{F}$ is $F$, it holds that

$$
\mu_{1}^{F}(K)=\int_{\mathrm{AGr}_{n-1}(V)} \chi(K \cap E) d m(E)
$$

for $K \in \mathcal{K}(V)$, where $m=\mathcal{C}^{-1} F$ is the Crofton measure of $\mu_{1}^{F}$, and we use the natural identification between smooth, translation-invariant measures on the affine Grassmannian $\operatorname{AGr}_{n-1}(V)$ with $C^{\infty}\left(\operatorname{Gr}_{n-1}(V)\right.$, Dens $\left.(V / E) \otimes|\omega|\right)$.

Recall that the generalized valuations $\mathcal{V}^{-\infty}(V)$ are the dual space to the smooth, compactly supported valuations $\mathcal{V}_{c}^{\infty}(V)$ equipped with the weak topology, see [3]. The representation of valuations through its pair of defining currents $(C, T)$ extends naturally to generalized valuations, except $C$ is now a distribution and $T$ a current, see [4]. Note that any (not necessarily compact) submanifold with corners $X \subset V$ defines an element $\chi_{X} \in \mathcal{V}^{-\infty}(V)$ through the evaluation map, and has $C\left(\chi_{X}\right)=\mathbb{1}_{X}$, $T(X)=\operatorname{nc}(X)$. 
By Alesker-Poincare duality we find that for any $\psi \in \mathcal{V}_{c}^{\infty}(V)$,

$$
\left\langle\mu_{1}^{F}, \psi\right\rangle=\int_{\mathrm{AGr}_{1}(V)} \psi(E) d m(E) .
$$

That is, $\mu_{1}^{F}=\int_{\mathrm{AGr}_{1}(V)} \chi_{E} d m(E)$, implying

$$
T\left(\mu_{1}^{F}\right)=\int_{\mathrm{AGr}_{1}(V)} T\left(\chi_{E}\right) d m(E)=\int_{\mathrm{AGr}_{1}(V)} \operatorname{nc}(E) d m(E) .
$$

Using the Euclidean structure to identify $m=C^{-1} F$ with an element of $C^{\infty}\left(S^{n-1}\right)$, and noting that $\mathrm{nc}\left(u^{\perp}+t u\right)=\llbracket\left(u^{\perp}+t u\right) \times\{ \pm u\} \rrbracket$, we conclude that

$$
\left\langle T\left(\mu_{1}^{F}\right), \eta\right\rangle=c_{n} \int_{\mathbb{R}^{n} \times S^{n-1}} C^{-1}(F) \alpha \wedge \operatorname{vol}_{S^{n-1}} \wedge \eta
$$

for every $\eta \in \Omega_{c}^{n-1}\left(\mathbb{R}^{n} \times S^{n-1}\right)$.

\section{Computing the Restriction}

4.1. The fiber integrals. Let $M \subset(V, F)$ be an immersed submanifold of dimension $m$. Recall $V=\mathbb{R}^{n}$ has a fixed Euclidean structure.

Fix a point $p \in M$ and local coordinates $\left(x^{0}, x^{1}, \ldots, x^{m-1}\right)$ for $M$ such that

$$
\left.\partial_{x^{i}}\right|_{p}=e_{i}, \quad i=0, \ldots, m-1
$$

for some orthonormal basis $e_{0}, e_{1}, \ldots, e_{n-1}$ of $V$.

Let us fix also a point $(p, u) \in S M$. Without loss of generality we may assume that $u=e_{0}$. We denote by $\widetilde{\partial}_{x^{i}}$ the horizontal lift of $\partial_{x^{i}}$ to $S M$ with respect to the induced Riemannian structure. Note that

$$
\widetilde{\partial}_{x^{0}}, \ldots, \widetilde{\partial}_{x^{m-1}}, e_{1}, \ldots, e_{m-1}
$$

form a basis of $T_{(p, u)} S M$. To construct these lifts, let $\gamma_{i}: I \rightarrow M$ be a smooth curve with $\gamma_{i}(0)=p$ and $\gamma_{i}^{\prime}(0)=\frac{\partial}{\partial x^{i}}$. Then

$$
\widetilde{\partial}_{x^{i}}=\left.\frac{d}{d t}\right|_{t=0} P_{\gamma} u(t)
$$

where $P_{\gamma} u$ is the curve in $S M$ defined via parallel transport of $u$ along $\gamma$.

We will also need the second fundamental form $h: T_{p} M \otimes T_{p} M \rightarrow T_{p} M^{\perp}$ of $M$,

$$
h(X, Y)=\left(\bar{\nabla}_{X} Y\right)^{\perp},
$$

where $\bar{\nabla}$ denotes the standard connection in $\mathbb{R}^{n}$. We write $h_{i j}=h\left(e_{i}, e_{j}\right)$, and $h_{i j}^{N}=\left\langle h_{i j}, N\right\rangle$.

Let $P_{m-1}^{k}$ be the set of increasing functions $\sigma:\{1, \ldots, k\} \rightarrow\{1, \ldots, m-1\}$. The complement is the obvious map $P_{m-1}^{k} \rightarrow P_{m-1}^{m-1-k}, \sigma \mapsto \sigma^{c}$.

Let $\theta_{0}, \ldots, \theta_{m-1}, \omega_{1,0}, \ldots, \omega_{m-1,0} \in T_{(p, u)}^{*} S M$ denote the basis dual to (66). By (44), the restrictred valuation $\left.\mu_{1}^{F}\right|_{M}$ has $T_{M}^{F}:=T\left(\left.\mu_{1}^{F}\right|_{M}\right)=\beta_{*} \bar{\alpha}^{*} T\left(\mu_{1}^{F}\right)$. As it is a vertical 
$m$-form, there are coefficients $A_{\sigma \tau}^{k}$ with $0 \leq k \leq m-1, \sigma \in P_{m-1}^{k}, \tau \in P_{m-1}^{m-1-k}$ such that

(7) $T_{M}^{F}=\theta_{0} \wedge \sum_{k=0}^{m-1} \sum_{\sigma \in P_{m-1}^{k}} \sum_{\tau \in P_{m-1}^{m-1-k}} A_{\sigma \tau}^{k} \theta_{\sigma(1)} \wedge \cdots \wedge \theta_{\sigma(k)} \wedge \omega_{\tau(1), 0} \wedge \cdots \wedge \omega_{\tau(m-1-k), 0}$.

Remark 4.1. More generally, the coefficients $A_{\sigma \tau}^{k}$ are naturally functions on the orthonormal frame bundle of $M$, that is, they are determined by the fixed immersion $M \leftrightarrow V$, the norm $F$, and the auxiliary Euclidean structure on $V$, which induces a Riemannian structure on $M$.

Proposition 4.2. It holds for all $k, \sigma, \tau$ that

$A_{\sigma \tau}^{k}=\epsilon c_{n} \int_{0}^{\pi / 2} \int_{S\left(T_{p} M^{\perp}\right)} \operatorname{det} h_{\sigma \tau^{c}}^{N}(p) \cos ^{m-k} \phi \sin ^{n-m-1+k} \phi \mathcal{C}^{-1} F(\cos \phi u+\sin \phi N) d N d \phi$, where $c_{n}$ is as in (5), and $\epsilon \in\{ \pm 1\}$ depends explicitly on $\sigma, \tau$.

Proof. As the form $T_{M}^{F}$ is smooth, we may ignore the blow-up construction of the restriction. Thus by Lemma 3.1, we have to compute the pushforward of $\mathcal{C}^{-1} F \alpha \wedge$ $\operatorname{vol}_{S^{n-1}}$ under the map $\beta:\left.S V\right|_{M} \backslash T M^{\perp} \rightarrow S M$.

Choose a smooth local section $N$ of the normal sphere bundle $S\left(T M^{\perp}\right)$. We define elements of $\left.T_{p, \cos \phi u+\sin \phi N_{p}} S V\right|_{M}$ by

$$
\begin{aligned}
\widehat{\partial}_{x^{i}} & =\left.\frac{d}{d t}\right|_{t=0}\left(\gamma_{i}(t), \cos \phi P_{\gamma_{i}} u+\sin \phi N \circ \gamma_{i}\right), \quad i=0, \ldots, m-1 \\
\hat{e}_{i} & =\left.\frac{d}{d t}\right|_{t=0}\left(p, \cos \phi\left(\cos t u+\sin t e_{i}\right)+\sin \phi N\right)=\left(0, \cos \phi e_{i}\right), \quad i=1, \ldots, m-1,
\end{aligned}
$$

where $\gamma_{i}: I \rightarrow M$ is a smooth curve with $\gamma_{i}(0)=p$ and $\gamma_{i}^{\prime}(0)=\frac{\partial}{\partial x^{i}}$. We may express the second coordinate $v_{i}$ of $\widehat{\partial}_{x^{i}}=\left(e_{i}, v_{i}\right)$ explicitly in terms of the second fundamental form, namely

$$
v_{i}=\cos \phi h_{0 i}+\sin \phi\left(-\sum_{j=0}^{m-1} h_{i j}^{N} e_{j}+\nabla_{e_{i}}^{\perp} N\right)
$$

where $\nabla^{\perp}$ denotes the normal connection. Indeed, if $\bar{\nabla}$ denotes the standard connection of the ambient space $V$, then for $0 \leq i \leq m-1$

$$
\left.\frac{d}{d t}\right|_{t=0}\left\langle P_{\gamma_{i}} u, e_{j}\right\rangle=\left\langle\bar{\nabla}_{e_{i}} P_{\gamma_{i}} u, e_{j}\right\rangle=\left\langle\nabla_{e_{i}} P_{\gamma_{i}} u, e_{j}\right\rangle=0, \quad j=0, \ldots, m-1,
$$

since for this range of indices, $e_{j}$ is tangent to $M$ at $p$. Since $e_{m}, \ldots, e_{n-1} \in T_{p} M^{\perp}$, by the definition of the second fundamental form, we have

$$
\left.\frac{d}{d t}\right|_{t=0}\left\langle P_{\gamma_{i}} u, e_{j}\right\rangle=\left\langle\bar{\nabla}_{e_{i}} P_{\gamma_{i}} u, e_{j}\right\rangle=\left\langle h_{0 i}, e_{j}\right\rangle, \quad j=m, \ldots, n-1 .
$$


To compute the constants $A_{\sigma \tau}^{k}$, we first note that

$$
e_{1}, \ldots, e_{m-1},-\sin \phi u+\cos \phi N, w_{1}, \ldots, w_{n-m-1}
$$

is an orthonormal basis of $T_{\cos \phi u+\sin \phi N} S^{n-1}$ if $N, w_{1}, \ldots, w_{n-m-1}$ is an orthonormal basis of $T_{p} M^{\perp}$, and

$$
\alpha\left(\widehat{\partial}_{x^{i}}\right)=\left\langle\cos \phi u+\sin \phi N, e_{i}\right\rangle= \begin{cases}\cos \phi, & i=0, \\ 0, & i=1, \ldots, m-1\end{cases}
$$

We integrate along the fiber, parametrized by $\gamma(\phi, N)=(p, \cos \phi u+\sin \phi N)$ so that $\frac{\partial}{\partial \phi} \gamma(\phi, N)=-\sin \phi u+\cos \phi N$. First, we compute

$$
\begin{aligned}
& B(\phi, N):=\alpha \wedge \operatorname{vol}_{S^{n-1}}\left(\widehat{\partial}_{x^{0}},\left(\widehat{\partial}_{x^{\sigma(j)}}\right)_{j=1}^{k},\left(\hat{e}_{\tau(j)}\right)_{j=1}^{m-1-k}, \frac{\partial}{\partial \phi} \gamma(\phi, N), w_{1}, \ldots, w_{n-m-1}\right) \\
& =\cos \phi \cdot \cos ^{m-1-k} \phi \cdot \operatorname{vol}_{S^{m-1}}\left(\left(v_{\sigma(j)}\right)_{j=1}^{k},\left(e_{\tau(j)}\right)_{j=1}^{m-1-k}\right) \\
& =(-1)^{k} \cos ^{m-k} \phi \sin ^{k} \phi \cdot \operatorname{vol}_{S^{m-1}}\left(\sum_{j=0}^{m-1} h_{\sigma(1), j}^{N} e_{j}, \ldots, \sum_{j=0}^{m-1} h_{\sigma(k), j}^{N} e_{j},\left(e_{\tau(j)}\right)_{j=1}^{m-1-k}\right) \\
& =\epsilon \cos ^{m-k} \phi \sin ^{k} \phi \operatorname{det} h_{\sigma \tau^{c}}^{N},
\end{aligned}
$$

for some $\epsilon \in\{ \pm 1\}$. This yields

$$
\begin{aligned}
A_{\sigma \tau}^{k} & =c_{n} \beta_{*} \bar{\alpha}^{*}\left(\mathcal{C}^{-1} F \cdot \alpha \wedge \operatorname{vol}_{S^{n-1}}\right)\left(\widetilde{\partial}_{x^{0}}, \widetilde{\partial}_{x^{\sigma(1)}}, \ldots, \widetilde{\partial}_{x^{\sigma(k)}}, e_{\tau(1)}, \ldots, e_{\tau(m-k-1)}\right) \\
& =c_{n} \int_{0}^{\pi / 2} \int_{S\left(T_{p} M^{\perp}\right)} \sin ^{n-m-1} \phi B(\phi, N) \mathcal{C}^{-1} F(\cos \phi u+\sin \phi N) d N d \phi \\
& =\epsilon c_{n} \int_{0}^{\pi / 2} \int_{S\left(T_{p} M^{\perp}\right)} \operatorname{det} h_{\sigma \tau^{c}}^{N}(p) \cos ^{m-k} \phi \sin ^{n-m-1+k} \phi \mathcal{C}^{-1} F(\cos \phi u+\sin \phi N) d N d \phi .
\end{aligned}
$$

The exact constant $c_{n}$ and sign $\epsilon$ will play no role in the following, and we omit them. In particular, we write

$$
\begin{gathered}
A^{0}=\int_{0}^{\pi / 2} \int_{S\left(T_{p} M^{\perp}\right)} \cos ^{m} \phi \sin ^{n-m-1} \phi \mathcal{C}^{-1} F(\cos \phi u+\sin \phi N) d N d \phi, \\
A_{\sigma \tau}^{1}=\left\langle h_{\sigma(1), \tau^{c}}, \int_{0}^{\pi / 2} \int_{S\left(T_{p} M^{\perp}\right)} \cos ^{m-1} \phi \sin ^{n-m} \phi N \mathcal{C}^{-1} F(\cos \phi u+\sin \phi N) d N d \phi\right\rangle .
\end{gathered}
$$

4.2. Simplifying the fiber integrals. Fix $S^{m-1} \subset S^{n-1}$. For almost every $v \in$ $S^{n-1}$ we may write $v=\cos \phi u+\sin \phi N$ with $u \in S^{m-1}, N \in S^{n-m-1}, \phi \in\left[0, \frac{\pi}{2}\right]$ in a unique way. For $f \in C^{\infty}\left(S^{n-1}\right), u \in S^{m-1}$ and $N \in S^{n-m-1}$ we will write

$$
\frac{\partial^{k} f}{\partial N^{k}}(u):=\left.\frac{d^{k}}{d \phi^{k}}\right|_{\phi=0} f(\cos \phi u+\sin \phi N) .
$$


More generally, if $\tilde{N}=\lambda N$ with $\lambda \in \mathbb{R}$, we write

$$
\frac{\partial^{k} f}{\partial \tilde{N}^{k}}(u)=\lambda^{k} \frac{\partial^{k} f}{\partial N^{k}}(u) \text {. }
$$

The coefficient $A^{0}$ is straightforward to simplify.

Lemma 4.3. $\left.A^{0}\right|_{S_{p} M}=\mathcal{C}_{m}^{-1}\left(\left.F\right|_{S_{p} M}\right)$.

Proof. Take $u^{\prime} \in S_{p} M$ and compute

$$
\begin{aligned}
& \mathcal{C}_{m} A^{0}\left(u^{\prime}\right) \\
& =\int_{0}^{\pi / 2} \int_{S_{p} M} \int_{S\left(T_{p} M^{\perp}\right)}\left|\left\langle u^{\prime}, u\right\rangle\right| \cos ^{m} \phi \sin ^{n-m-1} \phi \mathcal{C}_{n}^{-1} F(\cos \phi u+\sin \phi N) d N d u d \phi \\
& =\int_{0}^{\pi / 2} \int_{S_{p} M} \int_{S\left(T_{p} M^{\perp}\right)} \cos ^{m-1} \phi \sin ^{n-m-1} \phi\left|\left\langle u^{\prime}, v\right\rangle\right| \mathcal{C}_{n}^{-1} F(v) d N d u d \phi,
\end{aligned}
$$

where $v=\cos \phi u+\sin \phi N$ is the parametrization $v: S^{m-1} \times[0, \pi / 2] \times S^{n-m-1} \rightarrow$ $S^{n-1}$. Since $d v=\cos ^{m-1} \phi \sin ^{n-m-1} \phi d N d u d \phi$, we conclude that

$$
\mathcal{C}_{m} A^{0}\left(u^{\prime}\right)=\mathcal{C}_{n}\left(\mathcal{C}_{n}^{-1} F\right)\left(u^{\prime}\right)=F\left(u^{\prime}\right) .
$$

Corollary 4.4. The coefficient $A^{0}$ is determined by $\left.F\right|_{M}$.

As a warm-up for the general case, we compute $A_{\sigma \tau}^{1}$. We write $\left.\partial_{\phi}\right|_{\phi_{0}}$ for $\left.\frac{\partial}{\partial \phi}\right|_{\phi=\phi_{0}}$.

Lemma 4.5. For every even smooth function $f$ on $S^{n-1}, u \in S^{m-1}$, and $N \in$ $S^{n-m-1}$ we have

$$
\begin{gathered}
\int_{0}^{\pi / 2} \int_{S^{n-m-1}} \cos ^{m-1} \phi^{\prime} \sin ^{n-m} \phi^{\prime}\left\langle N, N^{\prime}\right\rangle f\left(\cos \phi u+\sin \phi N^{\prime}\right) d N^{\prime} d \phi^{\prime} \\
=\frac{1}{2} H^{-1}\left(\left.u^{\prime} \mapsto \partial_{\phi}\right|_{0} \mathcal{C} f\left(u^{\prime}, \phi, N\right)\right)(u),
\end{gathered}
$$

where here and in the following $(u, \phi, N)$ denotes the point $\cos \phi u+\sin \phi N \in S^{n-1}$.

Proof. The cosine transform can be written as

$\mathcal{C} f(u, \phi, N)$

$$
=\int_{S^{m-1}} \int_{S^{n-m-1}} \int_{0}^{\pi / 2} \cos ^{m-1} \phi^{\prime} \sin ^{n-m-1} \phi^{\prime}\left|K\left(u^{\prime}, \phi^{\prime}, N^{\prime}\right)\right| f\left(u^{\prime}, \phi^{\prime}, N^{\prime}\right) d \phi^{\prime} d N^{\prime} d u^{\prime},
$$

where

$$
K\left(u^{\prime}, \phi^{\prime}, N^{\prime}\right):=K\left(u, \phi, N, u^{\prime}, \phi^{\prime}, N^{\prime}\right):=\cos \phi \cos \phi^{\prime}\left\langle u, u^{\prime}\right\rangle+\sin \phi \sin \phi^{\prime}\left\langle N, N^{\prime}\right\rangle .
$$

Put $g_{\phi, u, N}\left(u^{\prime}, \phi^{\prime}, N^{\prime}\right)=\cos ^{m-1} \phi^{\prime} \sin ^{n-m-1} \phi^{\prime} K\left(u, \phi, N, u^{\prime}, \phi^{\prime}, N^{\prime}\right) f\left(u^{\prime}, \phi^{\prime}, N^{\prime}\right)$. We typically have $u, N$ fixed, and omit them from the notation. Let us compute

$$
\begin{aligned}
\left.\partial_{\phi}\right|_{0} \mathcal{C} f(u, \phi, N) & =\left.\partial_{\phi}\right|_{0}\left(\int_{\left(u^{\prime}, \phi^{\prime}, N^{\prime}\right): K(\phi) \geq 0} g_{\phi}-\int_{\left(u^{\prime}, \phi^{\prime}, N^{\prime}\right): K(\phi) \leq 0} g_{\phi}\right) \\
& =\left.2 \partial_{\phi}\right|_{0} \int_{\left(u^{\prime}, \phi^{\prime}, N^{\prime}\right): K(\phi) \geq 0} g_{\phi} .
\end{aligned}
$$


where the last equality follows by the symmetry $\left(u^{\prime}, \phi^{\prime}, N^{\prime}\right) \mapsto\left(-u^{\prime}, \phi^{\prime},-N^{\prime}\right)$, and the assumption that $f$ be even.

For any distribution $\eta$ on the sphere, $\left\langle\eta, g_{\phi}\right\rangle$ is by [26, Theorem 2.1.3] a smooth function of $\phi$ and $\partial_{\phi}\left\langle\eta, g_{\phi}\right\rangle=\left\langle\eta, \partial_{\phi} g_{\phi}\right\rangle$. The indicator function $\mathbb{1}_{K(\phi) \geq 0}$ regarded as a distribution on $S^{n-1}$ depending on the parameter $\phi$ is differentiable at $\phi=0$ and its derivative is a measure supported on $\left\{\left(u^{\prime}, \phi^{\prime}, N^{\prime}\right): K(0)=0\right\}$, see Eq. (15) below. By the chain rule,

$$
\left.\partial_{\phi}\right|_{0} \int_{K(\phi) \geq 0} g_{\phi}=\left.\partial_{\phi}\right|_{0}\left\langle g_{\phi}, \mathbb{1}_{K(\phi) \geq 0}\right\rangle=\left\langle\left.\partial_{\phi}\right|_{0} g_{\phi}, \mathbb{1}_{K(0) \geq 0}\right\rangle+\left\langle g_{0},\left.\partial_{\phi}\right|_{0} \mathbb{1}_{K(\phi) \geq 0}\right\rangle
$$

Since $g_{0}$ vanishes whenever $K(0)$ vanishes, the second summand is zero.

We conclude that

$$
\begin{array}{rl}
\left.\partial_{\phi}\right|_{0} \mathcal{C} & f(u, \phi, N)=\left.2 \int_{K(0) \geq 0} \partial_{\phi}\right|_{0} g_{\phi} \\
= & \int_{\left\langle u, u^{\prime}\right\rangle \geq 0} \int_{S^{n-m-1}} \int_{0}^{\pi / 2} \cos ^{m-1} \phi^{\prime} \sin ^{n-m} \phi^{\prime}\left\langle N, N^{\prime}\right\rangle f\left(u^{\prime}, \phi^{\prime}, N^{\prime}\right) d \phi^{\prime} d N^{\prime} d u^{\prime} \\
= & 2 H_{u^{\prime}}\left(\int_{S^{n-m-1}} \int_{0}^{\pi / 2} \cos ^{m-1} \phi^{\prime} \sin ^{n-m} \phi^{\prime}\left\langle N, N^{\prime}\right\rangle f\left(u^{\prime}, \phi^{\prime}, N^{\prime}\right) d \phi^{\prime} d N^{\prime}\right)(u),
\end{array}
$$

where $H_{u^{\prime}}$ denotes the hemispherical transform with respect to $u^{\prime} \in S^{m-1}$. Seeing that the argument of the hemispherical transform is odd in $u^{\prime}$, we may apply the inverse to conclude the proof.

Putting $\tilde{N}=h_{\sigma(1), \tau^{c}}$ and recalling $u=e_{0}$, we conclude, using notation (10), that

$$
A_{\sigma \tau}^{1}(u)=\frac{1}{2} H^{-1}\left(u^{\prime} \mapsto \frac{\partial F}{\partial h_{\sigma(1), \tau^{c}}}\left(u^{\prime}\right)\right)(u)
$$

For the general coefficient we do not obtain an explicit expression, but only some qualitative information. We first prove a lemma in harmonic analysis on the sphere.

Definition 4.6. We define the $\mathrm{SO}(m)$-equivariant transforms

$$
R_{D}^{k}, C_{D}^{k}: C^{\infty}\left(S^{m-1}\right) \rightarrow C^{\infty}\left(S^{m-1}\right)
$$

by

$$
R_{D}^{k} f(u)=\left.\int_{u^{\prime} \perp u} \frac{\partial^{k}}{\partial t^{k}}\right|_{t=0} f\left(\sqrt{1-t^{2}} u^{\prime}+t u\right) d u^{\prime}, \quad C_{D}^{k} f(u)=R_{D}^{k}(\langle u, \bullet\rangle f)(u)
$$

Lemma 4.7. Assume $m \geq 3$. Let $a_{k}, a_{k-2}, \ldots, a_{\epsilon} \geq 0$ with $a_{\epsilon} \neq 0, \epsilon \equiv k \bmod 2 \in$ $\{0,1\}$. Then

$$
T:=a_{k} R_{D}^{k}-a_{k-2} R_{D}^{k-2}+\cdots+(-1)^{\lfloor k / 2\rfloor} a_{\epsilon} R_{D}^{\epsilon}
$$

is a bijection on the space $C_{\epsilon}^{\infty}\left(S^{m-1}\right)$ of smooth functions on $S^{m-1}$ of parity $\epsilon$, and $T^{-1}$ is continuous. More precisely, there is an explicit constant $K=K(m)>0$ such that for every $q \geq 0$, the operator $T^{-1}: C_{\epsilon}^{q+K}\left(S^{m-1}\right) \rightarrow C_{\epsilon}^{q}\left(S^{m-1}\right)$ is continuous. 
Proof. As the transform commutes with $\mathrm{SO}(m), R_{D}^{k}$ is given by a scalar on the space of spherical harmonics of order $n$, denoted $\mathcal{H}_{n}^{m} \subset C^{\infty}\left(S^{m-1}\right)$. Let us first check that the eigenvalues of $R_{D}^{k}, R_{D}^{k+2}$ on $\mathcal{H}_{n}^{m}$ have opposite signs for every $k \geq 0$ of parity $\epsilon$, which would immediately imply the injectivity of $T$. Evaluating both on a zonal harmonic given by the Ledgendre polynomial $P_{n}^{m}\left(\left\langle u^{\prime}, e_{m}\right\rangle\right)$ with $n \equiv \epsilon \bmod 2$ and for $u=e_{m}$, we see that we ought to check

$$
\left(P_{n}^{m}\right)^{(k)}(0)\left(P_{n}^{m}\right)^{(k+2)}(0)<0
$$

It holds for $n \geq r$ that $\frac{d^{r}}{d t^{r}} P_{n}^{m}(t)=c P_{n-r}^{m+2 r}(t)$ for some $c>0$ [24, Lemma 3.3.9]. Taking $r=k, k+2$ and noting that $n^{\prime}=n-k$ is even, it remains to verify that for any $m^{\prime}$ and even $n^{\prime}$ we have

$$
P_{n^{\prime}}^{m^{\prime}}(0) P_{n^{\prime}-2}^{m^{\prime}+4}(0)<0 .
$$

Now sign $P_{n^{\prime}}^{m^{\prime}}(0)=(-1)^{n^{\prime} / 2}$ according to [24, Eq. (3.3.20)], and correspondingly $\operatorname{sign} P_{n^{\prime}-2}^{m^{\prime}+4}(0)=(-1)^{-1+n^{\prime} / 2}$. Thus $T$ is non-zero on every harmonic of parity $\epsilon$ and hence injective.

To prove that $T$ is surjective, let $\lambda_{n}$ be the eigenvalue of $T$ on $\mathcal{H}_{n}^{m}$, and $r_{n}$ the eigenvalue of $R_{D}^{\epsilon}$ on $\mathcal{H}_{n}^{m}$. Recall from [24, Eq. 3.1.15] that $\operatorname{dim} \mathcal{H}_{n}^{m}=: N(m, n)=$ $O\left(n^{m-2}\right)$ as $n \rightarrow \infty$. Letting $c_{j}$ denote various constants independent of $n$, we have by [24, Eq. (3.4.19)] for $l$ even $\left|P_{l}^{d}(0)\right|>c_{0} l^{-\frac{d-2}{2}}$ and hence by [24, Lemma 3.3.9]

$$
\left|r_{n}\right|=c_{1}\left|\left(P_{n}^{m}\right)^{(\epsilon)}(0)\right|=c_{2} \frac{N(m+2 \epsilon, n)}{N(m, n+\epsilon)}\left|P_{n-\epsilon}^{m+2 \epsilon}(0)\right| \geq c_{3} n^{-m / 2+\epsilon+1}
$$

As the signs in the definition of $T$ are alternating, it holds that $\left|\lambda_{n}\right| \geq\left|a_{\epsilon}\right|\left|r_{n}\right|$ and thus

$$
\left|1 / \lambda_{n}\right| \leq c_{4} n^{m / 2-\epsilon-1} .
$$

Take $g \in C_{\epsilon}^{\infty}\left(S^{m-1}\right)$, and let $g=\sum_{n \equiv \epsilon} g_{n}$ be its decomposition into spherical harmonics. Set $f:=\sum_{n \equiv \epsilon} \frac{1}{\lambda_{n}} g_{n}$. Then $f \in \bigcap_{s>0} H^{s}\left(S^{m-1}\right)=C^{\infty}\left(S^{m-1}\right)$ by (13) and $T f=g$. This proves that $T$ is surjective. Moreover, by the Sobolev embedding theorem (2), (13), and (11) we can for every $q \geq 0$ find $s, q^{\prime}=q+K(m)$ such that

$$
\|f\|_{C^{q}} \leq c_{5}\|g\|_{H^{s}} \leq c_{6}\|g\|_{C^{q^{\prime}}} .
$$

This proves the continuity of $T^{-1}$ and concludes the proof.

Proposition 4.8. For an even function $f \in C_{+}^{\infty}\left(S^{n-1}\right)$, point $u \in S^{m-1}$, and normal vector $N \in S^{n-m-1}$, define

$$
\begin{aligned}
& I_{k}^{n, m}(u, N ; f):= \\
& \int_{0}^{\pi / 2} \int_{S^{n-m-1}} \cos ^{m-k} \phi^{\prime} \sin ^{n-m-1+k} \phi^{\prime}\left\langle N, N^{\prime}\right\rangle^{k} f\left(\cos \phi u+\sin \phi N^{\prime}\right) d N^{\prime} d \phi^{\prime} .
\end{aligned}
$$

Then for any $q \geq 0$,

$$
\left\|I_{k}^{n, m}(\bullet, N ; f)\right\|_{C^{q}\left(S^{m-1}\right)} \leq c \sum_{j=0}^{k}\left\|\frac{\partial^{j}}{\partial N^{j}} \mathcal{C}_{n}(f)\right\|_{C^{L}\left(S^{m-1}\right)}
$$


for some constants $c=c(n, m, k, q)>0$, and $L=L(n, m, k, q) \in \mathbb{N}$.

Proof. As in the proof of Lemma 4.5 we write

$$
\begin{gathered}
K\left(u, \phi, N, u^{\prime}, \phi^{\prime}, N^{\prime}\right)=\cos \phi \cos \phi^{\prime}\left\langle u, u^{\prime}\right\rangle+\sin \phi \sin \phi^{\prime}\left\langle N, N^{\prime}\right\rangle, \\
g_{\phi}\left(u^{\prime}, \phi^{\prime}, N^{\prime}\right)=\cos ^{m-1} \phi^{\prime} \sin ^{n-m-1} \phi^{\prime} K\left(u, \phi, N, u^{\prime}, \phi^{\prime}, N^{\prime}\right) f\left(u^{\prime}, \phi^{\prime}, N^{\prime}\right) .
\end{gathered}
$$

We note that $\partial_{\phi}^{2} g_{\phi}=-g_{\phi}$, and record

$$
\begin{gathered}
g_{0}=\cos ^{m} \phi^{\prime} \sin ^{n-m-1} \phi^{\prime}\left\langle u, u^{\prime}\right\rangle f\left(u^{\prime}, \phi^{\prime}, N^{\prime}\right) \\
g_{0}^{\prime}:=\left.\partial_{\phi}\right|_{0} g_{\phi}=\cos ^{m-1} \phi^{\prime} \sin ^{n-m} \phi^{\prime}\left\langle N, N^{\prime}\right\rangle f\left(u^{\prime}, \phi^{\prime}, N^{\prime}\right)
\end{gathered}
$$

As in the proof of Lemma 4.5 we view $\mathbb{1}_{K(\phi) \geq 0}$ as a distribution on $S^{n-1}$ depending on the parameter $\phi$. The first and main technical step in the proof is to compute $\partial_{\phi}^{l} \mathbb{1}_{K(\phi) \geq 0}$ for any $l>0$. Note that the condition $K(\phi) \geq 0$ is equivalent to

$$
\left\langle u, u^{\prime}\right\rangle \geq-\tan \phi \tan \phi^{\prime}\left\langle N, N^{\prime}\right\rangle=: a(\phi)=a_{\phi^{\prime}, N^{\prime}}(\phi) .
$$

Putting $S_{u}^{m-2}=u^{\perp} \cap S^{m-1}$, we have for any function $h \in C^{\infty}\left(S^{m-1}\right)$

$$
\int_{\left\langle u, u^{\prime}\right\rangle \geq a(\phi)} h\left(u^{\prime}\right) d u^{\prime}=\int_{a(\phi)}^{1} \int_{S_{u}^{m-2}} h\left(\sqrt{1-t^{2}} v^{\prime}+t u\right){\sqrt{1-t^{2}}}^{m-3} d v^{\prime} d t .
$$

Since for any $\tilde{h} \in C^{\infty}(\mathbb{R})$

$$
\partial_{\phi} \int_{a(\phi)}^{1} \tilde{h}(t) d t=-\tilde{h}(a(\phi)) a^{\prime}(\phi),
$$

we conclude that for any smooth function $h\left(u^{\prime}, \phi^{\prime}, N^{\prime}\right)$ on $S^{n-1}$ parametrized by $S^{m-1} \times\left[0, \frac{\pi}{2}\right] \times S^{n-m-1}$,

$$
\partial_{\phi} \int_{u^{\prime}, \phi^{\prime}, N^{\prime}: K(\phi) \geq 0} h\left(u^{\prime}, \phi^{\prime}, N^{\prime}\right)
$$

$$
\begin{aligned}
& =\frac{1}{\cos ^{2} \phi} \int_{u^{\prime}, \phi^{\prime}, N^{\prime}: a_{\phi}\left(\phi^{\prime}, N^{\prime}\right)=\left\langle u, u^{\prime}\right\rangle} \tan \phi^{\prime}\left\langle N, N^{\prime}\right\rangle h\left(u^{\prime}, \phi^{\prime}, N^{\prime}\right) \\
& =\frac{1}{\cos ^{2} \phi} \int_{v^{\prime} \in S_{u}^{m-2}, \phi^{\prime}, N^{\prime}} \tan \phi^{\prime}\left\langle N, N^{\prime}\right\rangle h\left(\cos \theta v^{\prime}+\sin \theta u, \phi^{\prime}, N^{\prime}\right){\sqrt{1-a(\phi)^{2}}}^{m-3},
\end{aligned}
$$

where we use the auxiliary function $\theta=\theta\left(\phi^{\prime}, N^{\prime}\right)$ given by $\sin \theta=a_{\phi^{\prime}, N^{\prime}}(\phi)$.

Introduce

$$
\begin{aligned}
J_{r, s, t}^{\nu}(u, \phi, N ; h):=\tan ^{t} \phi \int_{v^{\prime}, \phi^{\prime}, N^{\prime}} \sqrt{1-a_{\phi^{\prime}, N^{\prime}}(\phi)^{2}} & \\
& \left\langle N, N^{\prime}\right\rangle^{\nu} \tan ^{\nu} \phi^{\prime} . \\
& \left.\cdot \partial_{z}^{r}\right|_{a(\phi)} h\left(\sqrt{1-z^{2}} v^{\prime}+z u, \phi^{\prime}, N^{\prime}\right) d v^{\prime} d N^{\prime} d \phi^{\prime} .
\end{aligned}
$$

Putting $\phi=0$, we find

$$
\left.J_{r, s, t}^{\nu}\right|_{\phi=0}=\left\{\begin{array}{cc}
J_{r}^{\nu}(u, N ; h), & t=0 \\
0, & t>0
\end{array}\right.
$$


where

$$
J_{r}^{\nu}(u, N ; h):=\left.\int_{u^{\prime} \perp u, \phi^{\prime}, N^{\prime}}\left\langle N, N^{\prime}\right\rangle^{\nu} \tan ^{\nu} \phi^{\prime} \partial_{z}^{r}\right|_{0} h\left(\sqrt{1-z^{2}} u^{\prime}+z u\right) d u^{\prime} d \phi^{\prime} d N^{\prime} .
$$

It follows directly from the definitions that

$$
\begin{aligned}
J_{r}^{k}\left(u, N ; g_{0}\right) & =C_{D}^{r}\left[I_{k}^{n, m}\left(u^{\prime}, N ; f\right)\right] ; \\
J_{r}^{k-1}\left(u, N ; g_{0}^{\prime}\right) & =R_{D}^{r}\left[I_{k}^{n, m}\left(u^{\prime}, N ; f\right)\right] .
\end{aligned}
$$

By eq. (15), we have

$$
\left\langle\partial_{\phi} \mathbb{1}_{K(\phi) \geq 0}, h\right\rangle=J_{0, m-3,0}^{1}+J_{0, m-3,2}^{1}
$$

and it is easy to verify that

(18) $\partial_{\phi} J_{r, s, t}^{\nu}=t\left(J_{r, s, t-1}^{\nu}+J_{r, s, t+1}^{\nu}\right)-s\left(J_{r, s-2, t+1}^{\nu+2}+J_{r, s-2, t+3}^{\nu+2}\right)-\left(J_{r+1, s, t}^{\nu+1}+J_{r+1, s, t+2}^{\nu+1}\right)$

The value of $\left\langle\left.\partial_{\phi}^{l}\right|_{0} \mathbb{1}_{K(\phi) \geq 0}, h\right\rangle$ will thus be a linear combination of various $J_{r}^{\nu}(u, N ; h)$. We think of the end formula as the result of $(l-1)$ differentiations applied to each of the two summands in (17), the sum at each step consisting of summands $J_{r, s, t}^{\nu}$, splitting into six summands according to (18). In the last step, all summands with $t>0$ vanish. Each sequence of splittings is referred to as a path. The combinatorics is illustrated in the following diagram.

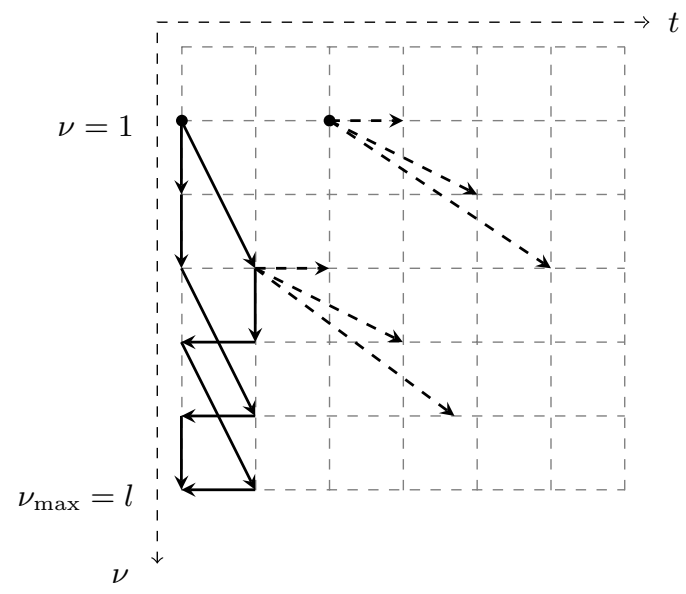

Figure 1. The two dots represent the two summands in (17). Each arrow represents a splitting. The two solid black paths only ever pass through the first summand in each bracket in (18). The dashed arrows represent some splittings given by the second summands in each bracket.

It follows immediately from (18) that any $J_{r}^{\nu}$ appearing in the final linear combination will have $r<\nu$ and $r \equiv \nu+1 \bmod 2$. It is also easy to see that the maximal $\nu$ appearing is $\nu_{\max }=l$. We thus write

$$
\left\langle\left.\partial_{\phi}^{l}\right|_{0} \mathbb{1}_{K(\phi) \geq 0}, h\right\rangle=\sum_{r^{\prime}=1}^{\lfloor(l+1) / 2\rfloor} a_{l+1-2 r^{\prime}} J_{l+1-2 r^{\prime}}^{l}(u, N ; h)+\sum_{r<\nu<l} a_{\nu, r} J_{r}^{\nu}(u, N ; h)
$$


This completes the first and main technical step in the proof.

We will now prove the proposition by induction on $k$. The statement for $k=0,1$ follows from Lemmas 4.3 and 4.5. Suppose that it has been proved for $k^{\prime}<k$. In the following, $h$ is either $g_{0}$ or $g_{0}^{\prime}$, and $l=k-1$. By the induction assumption, it holds for all $k^{\prime}<k$ and all $q^{\prime}$ that

$$
\left\|I_{k^{\prime}}^{n, m}(\bullet, N ; f)\right\|_{C^{q^{\prime}\left(S^{m-1}\right)}} \leq c^{\prime} \sum_{j=0}^{k^{\prime}}\left\|\frac{\partial^{j}}{\partial N^{j}} C f\right\|_{C^{L^{\prime}\left(S^{m-1}\right)}}
$$

for $c^{\prime}=c\left(n, m, k^{\prime}, q^{\prime}\right)$ and $L^{\prime}=L\left(n, m, k^{\prime}, q^{\prime}\right)$. It follows by (16) and from the continuity of $R_{D}^{r}, C_{D}^{r}$ in the appropriate topologies, that $J_{r}^{\nu}(u, N ; h)$ for $h=g_{0}$ and $\nu \leq k-1$, as well as for $h=g_{0}^{\prime}$ and $\nu \leq k-2$, satisfy

$$
\left\|J_{r}^{\nu}(\bullet, N ; h)\right\|_{C^{q^{\prime}}\left(S^{m-1}\right)} \leq \tilde{c} \sum_{j=0}^{k^{\prime}}\left\|\frac{\partial^{j}}{\partial N^{j}} \mathcal{C} f\right\|_{C^{\tilde{L}}\left(S^{m-1}\right)}
$$

for certain $\tilde{c}=\tilde{c}\left(n, m, k^{\prime}, q^{\prime}\right)$ and $\tilde{L}=\tilde{L}\left(n, m, k^{\prime}, q^{\prime}\right)$. This applies to all summands in the second sum of (19) as we assume $l=k-1$.

To understand the first sum in (19), we can ignore the second summand in each bracket in (18), as well as the second summand in (17), since any endpoint $J_{r}^{\nu}$ of a path of length $l-1$ passing through either of the second summands, that ends with $t=0$, must have $\nu \leq l-2$, which lands it in the second sum of (19).

We thus consider the principal term recursion

$$
\partial_{\phi} J_{r, s, t}^{\nu} \equiv t J_{r, s, t-1}^{\nu}-s J_{r, s-2, t+1}^{\nu+2}-J_{r+1, s-1, t}^{\nu+1}
$$

Differentiate the first summand in eq. (17) $l-1$ times using this recursion. We look at paths that pass $\alpha_{j}$ times in the $j$ th summand of (18), $j=1,2,3$, with $\alpha_{1}+\alpha_{2}+\alpha_{3}=l-1$. We start with $t=0$, and must have $t=0$ in the last step, so that $\alpha_{1}=\alpha_{2}$, in particular $\alpha_{3} \equiv l-1 \bmod 2$. The path contributes towards the coefficient $a_{r}$ of $J_{r}^{l}$ with $r=\alpha_{3}$, and the sign of each contribution is $(-1)^{\alpha_{2}+\alpha_{3}}=(-1)^{\frac{k-2-r}{2}+r}=(-1)^{\frac{k-2+r}{2}}$. Since for any $r \leq k-2$ of parity $k \bmod 2$ there are non-zero contributions, $\operatorname{sign} a_{r}=(-1)^{\frac{k-2+r}{2}}$.

Now recall from the proof of Lemma 4.5 that by symmetry,

$$
\mathcal{C} f(u, \phi, N)=\left\langle g_{\phi}, \mathbb{1}_{K(\phi) \geq 0}\right\rangle-\left\langle g_{\phi}, \mathbb{1}_{K(\phi) \leq 0}\right\rangle=2\left\langle g_{\phi}, \mathbb{1}_{K(\phi) \geq 0}\right\rangle
$$

Note that $\partial_{\phi}^{i} \mathbb{1}_{K(\phi) \geq 0}$ is by (15) supported on $\{K(\phi)=0\}$, while $g_{\phi}$ vanishes on $\{K(\phi)=0\}$. Note also that $\partial_{\phi} \mathbb{1}_{K(\phi) \geq 0}$ is a Borel measure. Hence

$$
\frac{1}{2} \partial_{\phi} \mathcal{C} f(u, \phi, N)=\left\langle\partial_{\phi} g_{\phi}, \mathbb{1}_{K(\phi) \geq 0}\right\rangle
$$

and 


$$
\begin{aligned}
\frac{1}{2} \partial_{\phi}^{p} \mathcal{C} f(u, \phi, N) & =\sum_{i=1}^{p}\left(\begin{array}{c}
p-1 \\
i-1
\end{array}\right)\left\langle\left.\partial_{\phi}^{i}\right|_{0} g_{\phi},\left.\partial_{\phi}^{p-i}\right|_{0} \mathbb{1}_{K(\phi) \geq 0}\right\rangle \\
& =\sum_{i=1}^{\lfloor p / 2\rfloor}\left(\begin{array}{c}
p-1 \\
2 i-1
\end{array}\right)(-1)^{i}\left\langle g_{0},\left.\partial_{\phi}^{p-2 i}\right|_{0} \mathbb{1}_{K(\phi) \geq 0}\right\rangle \\
& +\sum_{i=0}^{\lfloor(p-1) / 2\rfloor}\left(\begin{array}{c}
p-1 \\
2 i
\end{array}\right)(-1)^{i}\left\langle g_{0}^{\prime},\left.\partial_{\phi}^{p-2 i-1}\right|_{0} \mathbb{1}_{K(\phi) \geq 0}\right\rangle
\end{aligned}
$$

Rewrite eq. (21) with $p=k$ as

$$
\begin{aligned}
\left\langle g_{0}^{\prime},\left.\partial_{\phi}^{k-1}\right|_{0} \mathbb{1}_{K(\phi) \geq 0}\right\rangle=\frac{1}{2} \partial_{\phi}^{k} \mathcal{C} f(u, \phi, N) & -\sum_{i=1}^{k / 2}\left(\begin{array}{c}
k-1 \\
2 i-1
\end{array}\right)(-1)^{i}\left\langle g_{0},\left.\partial_{\phi}^{k-2 i}\right|_{0} \mathbb{1}_{K(\phi) \geq 0}\right\rangle \\
& -\sum_{i=1}^{(k-1) / 2}\left(\begin{array}{c}
k-1 \\
2 i
\end{array}\right)(-1)^{i}\left\langle g_{0}^{\prime},\left.\partial_{\phi}^{k-2 i-1}\right|_{0} \mathbb{1}_{K(\phi) \geq 0}\right\rangle
\end{aligned}
$$

By equations (19) and (20), we see that for some $c_{1}=c_{1}\left(n, m, k, q^{\prime}\right)$,

$$
\left\|\left\langle g_{0}^{\prime},\left.\partial_{\phi}^{k-1}\right|_{0} \mathbb{1}_{K(\phi) \geq 0}\right\rangle\right\|_{C^{q^{\prime}\left(S^{m-1}\right)}} \leq c_{1} \sum_{j=0}^{k}\left\|\frac{\partial^{j}}{\partial N^{j}} \mathcal{C} f\right\|_{C^{\tilde{L}}\left(S^{m-1}\right)} .
$$

Now set $H(u):=I_{k}^{n, m}(u, N ; f)$. Putting $\epsilon=k \bmod 2$, we note that $H \in$ $C^{\infty}\left(S^{m-1}\right)$ has parity $\epsilon$. Define

$$
T=\sum_{r^{\prime}=1}^{k / 2} a_{k-2 r^{\prime}} R_{D}^{k-2 r^{\prime}}: C^{\infty}\left(S^{m-1}\right) \rightarrow C^{\infty}\left(S^{m-1}\right),
$$

with the coefficients from the first sum of (19). We have by eq. (16)

$$
T(H)=\sum_{r^{\prime}=1}^{k / 2} a_{k-2 r^{\prime}} J_{k-2 r^{\prime}}^{k-1}\left(u, N ; g_{0}^{\prime}\right)
$$

and so by eq. (19) with $l=k-1$, together with eqs. (22) and (20) we have

$$
\|T(H)\|_{C^{q^{\prime}\left(S^{m-1}\right)}} \leq c_{2} \sum_{j=0}^{k}\left\|\frac{\partial^{j}}{\partial N^{j}} \mathcal{C} f\right\|_{C^{\tilde{L}}\left(S^{m-1}\right)}
$$

Since the coefficients $a_{k-2 r^{\prime}}$ have alternating signs, by Lemma 4.7 we know that $T^{-1}$ : $C_{\epsilon}^{q^{\prime}}\left(S^{m-1}\right) \rightarrow C_{\epsilon}^{q}\left(S^{m-1}\right)$ is continuous for $q^{\prime}=q+K(m)$. Taking $L(n, m, k, q)=$ $\tilde{L}\left(n, m, k-1, q^{\prime}\right)$ and $c(n, m, k, q)=c_{2}$ concludes the proof.

Observe that we can view the manifold of ordered orthonormal bases in the tangent spaces to $M$ also as the total space of a principal bundle over the sphere bundle, with the bundle projection $\pi_{0}: F M \rightarrow S M, \pi_{0}\left(u_{0}, \ldots, u_{n-1}\right)=u_{0}$. After a choice of local section $b: U \rightarrow F M$, the coefficients $A_{\sigma \tau}^{k}=A_{\sigma \tau}^{k}(\bullet ; b)$ are well-defined functions on $U \subset S M$. 
Corollary 4.9. The coefficients $A_{\sigma \tau}^{k}$ computed in subsection 4.1 are determined at $p \in M$ by the restriction to $S_{p} M$ of all derivatives $\frac{\partial^{i} F}{\partial N^{i}}, i \leq k$, in all directions $N \in$ Image $\left(h_{p}\right)$. Moreover, there are constants $L=L(n, m, k)$ and $c=c(n, m, k)$ such that for any choice of local section $b: U \rightarrow F M$

$$
\begin{aligned}
& \left\|A_{\sigma \tau}^{k}(\bullet ; b)\right\|_{C^{0}\left(S_{p} M \cap U\right)} \\
& \quad \leq c \sup _{v_{1}, \ldots, v_{2 k} \in S_{p} M} \sum_{j=0}^{k}\left\|h_{p}\right\|_{\infty}^{k-j}\left\|\frac{\partial^{j} F}{\partial\left(h_{p}\left(v_{1}, v_{2}\right)+\cdots+h_{p}\left(v_{2 k-1}, v_{2 k}\right)\right)^{j}}\right\|_{C^{L}\left(S_{p} M\right)}
\end{aligned}
$$

Proof. In light of Proposition 4.8 and using eq. (8), it only remains to express the determinants $\operatorname{det} h_{\sigma \tau^{c}}^{N^{\prime}}(p)$ for $N \in S\left(T_{p} M^{\perp}\right)$ as linear combinations

$$
\operatorname{det} h_{\sigma \tau^{c}}^{N^{\prime}}(p)=\sum c_{j}\left\langle H_{j}, N^{\prime}\right\rangle^{k}
$$

for some $c_{j} \in \mathbb{R}$ and $H_{j} \in \operatorname{Image}\left(h_{p}\right)$. As

$$
2^{k} k ! x_{1} \cdots x_{k}=\sum_{\epsilon \in\{1,-1\}} \epsilon_{1} \cdots \epsilon_{k}\left(\epsilon_{1} x_{1}+\cdots+\epsilon_{k} x_{k}\right)^{k}
$$

we see that the products $\left\langle h_{\sigma\left(i_{1}\right), \tau^{c}(1)}, N^{\prime}\right\rangle \cdots\left\langle h_{\sigma\left(i_{k}\right), \tau^{c}(k)}, N^{\prime}\right\rangle$ arising in the determinant are given by universal linear combinations of monomials of the form

$$
\left\langle\epsilon_{1} h_{\sigma\left(i_{1}\right), \tau^{c}(1)}+\cdots+\epsilon_{k} h_{\sigma\left(i_{k}\right), \tau^{c}(k)}, N^{\prime}\right\rangle^{k}
$$

with $\epsilon_{j} \in\{1,-1\}$. This proves the first part. Proposition 4.8 with $q=0$ readily gives the desired bound on the norm.

Proof of Theorem $C$. The statement is contained in the first part of Corollary 4.9.

Remark 4.10. The function $F \in C^{\infty}\left(S^{n-1}\right)$ need not be the restriction of a norm; formula (8) and Corollary 4.9 apply to arbitrary smooth $F$. Observe also that $A_{\sigma \tau}^{k}$ depends linearly on $F$.

\section{The WEAK WeYl PRINCIPLE}

5.1. Geometric preliminaries. Recall the tautological map $\theta: S M \rightarrow S(V)$, $\theta(x, v)=v$. The second fundamental form of $M$ is $h: \operatorname{Sym}^{2}(T M) \rightarrow T M^{\perp}$, $h(X, Y)=\left(\bar{\nabla}_{X} Y\right)^{\perp}$.

Lemma 5.1. Image $\left(d_{p, u} \theta\right)=T_{u} \theta\left(S_{p} M\right) \oplus \operatorname{Image}\left(h_{p}(u, \bullet)\right)$.

Proof. In one direction, first observe that $T_{u} \theta\left(S_{p} M\right) \subset \operatorname{Image}\left(d_{p, v} \theta\right)$. If $v_{0} \in T_{p} M$ is nonzero, $p(t)$ is a curve through $p$ with $p^{\prime}(0)=v_{0}$ and $u(t)$ is the parallel transport of $u$ along $p(t)$, then putting $\gamma(t)=(p(t), u(t))$ we find

$$
h_{p}\left(u, v_{0}\right)=u^{\prime}(0)=d_{p, u} \theta\left(\gamma^{\prime}(0)\right) .
$$

Thus Image $\left(h_{p}(u, \bullet)\right) \subset$ Image $\left(d_{p, u} \theta\right)$. In the other direction, let $\gamma(t)=(p(t), u(t))$ be arbitrary with $u(t) \in S M$ and $\gamma(0)=(p, u)$. Then $d \theta\left(\gamma^{\prime}(0)\right)=u^{\prime}(0)=$ $h_{p}\left(u, p^{\prime}(0)\right)+\nabla_{p^{\prime}(0)} u(t)$. It remains to note that $\nabla_{p^{\prime}(0)} u(t) \in T_{p} M$, and $\left\langle\nabla_{p^{\prime}(0)} u(t), u\right\rangle=$ 0 . 
Corollary 5.2. $M$ is directionally regular at $(p, u) \in S M$ if and only $h_{p}(u, \bullet)$ has rank $\min (m, n-m)$.

Proof. Follows immediately from Lemma 5.1,

We now show that most immersions are directionally regular at most points. Equip $C^{\infty}(M, V)$ with the smooth Whitney topology, see [23] for details. A residual subset is the countable intersection of open dense subsets.

Lemma 5.3. Let $W \subset C^{\infty}(M, V)$ be the subset of smooth immersions of the manifold $M^{m}$ in the linear space $V^{n}$. Then there is a dense residual subset $W^{\prime} \subset W$ such that for all $f \in W^{\prime}, f$ is directionally regular on a dense open subset of $S M$.

Proof. For basic notions of jet bundles of smooth functions, we refer to [23]. By 23. Lemma 5.5], $W$ is an open subset of $C^{\infty}(M, V)$.

For $f \in C^{\infty}(M, V)$, let $\tilde{\theta}_{f}: T M \rightarrow V$ be the composition of $d f: T M \rightarrow T V$ with the projection to the second factor of $T V=V \times V$, which is just the previously encountered tautological map, extended to arbitrary smooth maps.

Denote $k=\min \{n, 2 m\}$. For $(q, u) \in T M, u \neq 0$, define the subset $W_{q, u}:=$ $\left\{j_{q}^{2} f: \operatorname{rank}\left(d_{q, u} \tilde{\theta}_{f}\right)<k\right\}$ of the bundle of 2-jets $J^{2}(M, V)$. Note that $W_{q, u}$ is a finite union of locally closed submanifolds, each of codimension at least $m+1$. By Thom's transversality theorem [23, Theorem 4.9], each set $\left\{f \in C^{\infty}(M, V): j^{2} f\right.$ 币 $\left.W_{q, u}\right\}$ is residual in $C^{\infty}(M, V)$. Note that $j^{2} f(M) \subset J^{2}(M, V)$ is an $m$-dimensional submanifold, and therefore $j^{2} f \pitchfork W_{q, u} \Longleftrightarrow j^{2} f(M) \cap W_{q, u}=\emptyset$. Now choose a dense sequence $\left(q_{i}, u_{i}\right) \in T M \backslash \underline{0}$. By [23, Lemma 3.3], any residual subset of $C^{\infty}(M, V)$ is dense, and it follows that the intersection

$$
W^{\prime}:=W \cap \bigcap_{i \geq 1}\left\{f \in C^{\infty}(M, V): j^{2} f(M) \cap W_{q_{i}, u_{i}}=\emptyset\right\}
$$

is a dense residual subset of $W$. Finally, note that the set of directionally regular points of any given immersion is open. Thus any $f \in W^{\prime}$ is directionally regular at an open subset containing all $\left(q_{i}, u_{i}\right)$, concluding the proof.

5.2. When WWP fails. As before, in the following $M \subset V$ is an immersed submanifold, $m=\operatorname{dim} M$ and $n=\operatorname{dim} V$. We first prove Theorem $\mathrm{B}$, which is implied by the following two statements.

Lemma 5.4. Consider the hemispherical transform $H: C^{\infty}\left(S^{m-1}\right) \rightarrow C^{\infty}\left(S^{m-1}\right)$, and let $a: S^{m-1} \rightarrow S^{m-1}$ be the antipodal map. Assume $u, v \in S^{m-1}$ and $u \perp v$. Let $U$ be any neighborhood of $u$. Then there is an odd function $f \in C_{c}^{\infty}(U \cup a U)$ such that $H^{-1}(f)(v)=1$.

Proof. Let $\tilde{f}$ be a smooth, odd, $\operatorname{Stab}(u)=\mathrm{SO}(m-1)$-invariant function supported in $U \cup a U$ with $d_{u} \tilde{f} \neq 0$. Set $\tilde{g}=H^{-1} \tilde{f}$, which is clearly $\mathrm{SO}(m-1)$-invariant. Note that the hypersphere $u^{\perp}$ must lie entirely in the support of $\tilde{g}$, otherwise by $\mathrm{SO}(m-1)$-invariance there is an open band around $u^{\perp}$ where $\tilde{g}$ vanishes, but then $\tilde{f}=H \tilde{g}$ must be constant in a neighborhood of $u$. We may then find a small rotation $R \in \mathrm{SO}(m)$ such that $R \tilde{f}$ is supported in $U \cup a U$, and $H^{-1}(R \tilde{f})(v)=R \tilde{g}(v) \neq 0$. It remains to set $f=\frac{1}{R \tilde{g}(v)} R \tilde{f}$. 
Proposition 5.5. Assume $m=\operatorname{dim} M \geq 3$, and $\theta: S M \rightarrow S^{n-1}$ has constant rank $r \leq n-2$ near $(p, u) \in S M$. Assume moreover that $\operatorname{Image}\left(h_{p}\right) \neq \operatorname{Image}\left(h_{p}(u, \bullet)\right)$. Then there is a neighborhood $U \subset M$ of $p$ such that the coefficients $A_{\sigma \tau}^{1}$ at $(p, u)$ are not determined by $\left.F\right|_{U}$.

Proof. Since Image $\left(h_{p}\right) \neq \operatorname{Image}\left(h_{p}(u, \bullet)\right)$, we can find $e_{1} \in S_{p} M, e_{1} \perp u$ with $h_{p}\left(e_{1}, e_{1}\right) \notin \operatorname{Image}\left(h_{p}(u, \bullet)\right)$. Fix some $e_{0} \in S_{p} M$ such that $e_{0} \perp e_{1}, u$. Then $A_{\sigma \tau}^{1}\left(e_{0}\right)=\frac{1}{2} H^{-1}\left(\left.\partial_{\phi}\right|_{0} F\left(u^{\prime}, \phi, h_{11}\right)\right)\left(e_{0}\right)$ with $\sigma=\{1\}, \tau=\{2, \ldots, m-1\}$ by eq. (12).

As $\theta$ has constant rank near $(p, u)$, the same is by Lemma 5.1 true near $(p,-u)$. Hence there are open neighborhoods $U \subset M$ of $p$ and $W \subset S^{n-1}$ of $u$ such that $Z:=\theta(S U) \cap(W \cup a W) \subset S^{n-1}$ is an embedded submanifold, and $T_{u} Z=$ $T_{u} \theta\left(S_{p} M\right) \oplus \operatorname{Image}\left(h_{p}(u, \bullet)\right)$. As $h_{11}=h_{p}\left(e_{1}, e_{1}\right)$ is transversal to $Z$ at $u$, we see that $u^{\prime} \mapsto \frac{\partial}{\partial h_{11}} F\left(u^{\prime}\right), u^{\prime} \in S_{p} M$, can be perturbed arbitrarily in a small neighborhood of $\{u,-u\} \subset S_{p} M$ by perturbing $F$, while keeping $\left.F\right|_{\theta(S U)}$ fixed and $F$ symmetric. Such a perturbation, if small enough, will not change the convexity of $F$, but by Lemma 5.4, $H^{-1}\left(u^{\prime} \mapsto \frac{\partial}{\partial h_{11}} F\left(u^{\prime}\right)\right)\left(e_{0}\right)$ will not be determined by $\left.F\right|_{\theta(S U)}$.

Proof of Theorem $B$. By directional regularity, $\theta$ has constant rank in a neighborhood of $(p, u)$. Clearly, $\operatorname{dim} \operatorname{Image}\left(h_{p}(u, \bullet)\right) \leq m$. Hence if $\operatorname{dim} \mathcal{O}_{p}^{2} M>2 m$, then Image $\left(h_{p}\right) \neq \operatorname{Image}\left(h_{p}(u, \bullet)\right)$. The statement follows now from Proposition 5.5

When $m+3 \leq n \leq 2 m$, WWP generically holds by Theorem $\mathrm{A}$, but can fail for particular submanifolds, as the following examples show.

Example 5.6. Consider a generic surface $\Sigma^{2} \subset \mathbb{R}^{5}$, which has dim Image $h_{q}^{\Sigma}=3$ for all $q \in \Sigma$, and $\operatorname{dim} \operatorname{Image} h_{q}^{\Sigma}(v, \bullet)=2$ for $(q, v)$ in an open dense subset of $S \Sigma$. Now take $M=\Sigma \times \mathbb{R} \subset \mathbb{R}^{6}$. Writing $u=(v, \xi) \in T_{q, s} M=T_{p} M \oplus \mathbb{R}$, we have $h_{q, s}\left(u_{1}, u_{2}\right)=$ $h_{q}^{\Sigma}\left(v_{1}, v_{2}\right)$. Hence dim Image $h_{p}=3$ everywhere, while dim Image $h_{p}(u, \bullet)=2$ for $(p, u)$ in an open and dense subset of $S M$. Fixing such $(p, u), d \theta$ has constant rank 4 in its neighborhood, and Proposition 5.5 shows that WWP fails. Note that by taking $M=\Sigma \times \mathbb{R}^{k}$ we can produce higher dimensional examples of codimension 3 for which WWP fails.

Example 5.7. For a less trivial example, let $M^{4} \subset \mathbb{R}^{8}$ be given by

$$
M=\left\{\left(x_{1}, x_{2}, x_{3}, x_{4}, x_{1} x_{3}, x_{1} x_{4}, x_{2} x_{3}, x_{2} x_{4}\right)\right\},
$$

which is nothing but the Segré embedding $\mathbb{P}^{2} \times \mathbb{P}^{2} \hookrightarrow \mathbb{P}^{8}$ in affine charts. The second fundamental form can be represented in a certain frame of the normal bundle by

$$
h_{x}(u, v)=\left(u_{1} v_{3}+u_{3} v_{1}, u_{1} v_{4}+u_{4} v_{1}, u_{2} v_{3}+u_{3} v_{2}, u_{2} v_{4}+u_{4} v_{2}\right) .
$$

Hence dim Image $h_{x}=4$ everywhere, while dim Image $h_{x}(u, \bullet)=3$ for generic $u$, and so Proposition 5.5 can be applied to deduce that WWP fails for $M$. By taking products with $\mathbb{R}^{k}$ we can promote $M$ to higher dimensional examples of codimension 4 for which WWP fails. 
5.3. When WWP holds. We now turn to the positive results. The weak Weyl principle ultimately follows from the relationship between the derivatives of $F$ along the tangent and normal directions to the manifold.

Proposition 5.8. Assume $M^{m} \subset V^{n}$, and $\theta: S M \rightarrow S^{n-1}$ is submersive in a dense subset of $(p, v) \in S M$. Then the coefficients $A_{\sigma \tau}^{k}(p, u ; F)$ on $S_{p} M$ are determined by $\left(M,\left.F\right|_{M}\right)$ for all $k \geq 0$.

Proof. By assumption, we have Image $\left(h_{p}\right)=\operatorname{Image}\left(h_{p}(v, \bullet)\right)=T_{p} M^{\perp}$ for $(p, v)$ in a dense subset $D \subset S M$. It suffices by Corollary 4.9 to show that for any $u \in S_{p} M$, and any $N \in T_{p} M^{\perp}, \frac{\partial^{k} F}{\partial N^{k}}(u)$ is determined by $\left.F\right|_{\theta(S M)}$.

Assume first that $(p, u) \in D$. Then $\theta(S M) \subset S^{n-1}$ contains a neighborhood of $u$ in $S^{n-1}$, concluding this case. For general $(p, u) \in S M$ and $N \in T_{p} M^{\perp}$, we choose sequences $D \ni\left(p_{j}, u_{j}\right) \rightarrow(p, u), T_{p_{j}} M^{\perp} \ni N_{j} \rightarrow N$, and obtain $\frac{\partial^{k} F}{\partial N^{k}}(u)=$ $\lim _{j \rightarrow \infty} \frac{\partial^{k} F}{\partial N_{j}^{k}}\left(u_{j}\right)$.

Lemma 5.9. Assume $M^{m} \subset V^{n}$, and Image $\left(h_{p}\right)=\operatorname{Image}\left(h_{p}(u, \bullet)\right)$ in a dense subset of $(p, u) \in S M$. Then the coefficients $A_{\sigma \tau}^{1}(p, u ; F)$ on $S_{p} M$ are determined by $\left(M,\left.F\right|_{M}\right)$.

Proof. Consider first $(p, u)$ in the given dense set $D \subset S M$. Then for any $N \in$ Image $\left(h_{p}\right)$ we can find $v \in T_{p} M$ such that $h_{p}(u, v)=N$. Choose a curve $\gamma(t)$ with $\gamma(0)=p, \gamma^{\prime}(0)=v$, and let $u(t)$ be the parallel transport of $u$ along $\gamma(t)$. It follows that

$$
\frac{\partial F}{\partial N}(u)=\left.\frac{d}{d t}\right|_{t=0} F(u(t)) .
$$

is determined by $\left.F\right|_{M}$. We conclude the proof by continuity as in Proposition 5.8,

Lemma 5.10. Assume $M^{m} \subset V^{n}$ and $n=m+2$. If dim Image $h_{y}=1$ in some open neighborhood of $p \in M$, then either a neighborhood of $p$ in $M$ lies in a hyperplane, or $h_{p}: T_{p} M \times T_{p} M \rightarrow \operatorname{Image}\left(h_{p}\right)$ is a symmetric form of rank one.

Proof. We may assume $p=0$, and $M$ is the graph of $F=(f, g): \mathbb{R}^{m} \rightarrow \mathbb{R}^{2}$, with $\nabla f(0)=\nabla g(0)=0$. Denote $\bar{F}(x)=(x, F(x)): \mathbb{R}^{m} \rightarrow \mathbb{R}^{m+2}$, and for $v \in \mathbb{R}^{m}$ write

$$
\bar{v}=\left.\bar{F}_{*} v\right|_{x}=\left(v, \frac{\partial F}{\partial v}(x)\right) \in T_{\bar{F}(x)} M .
$$

Observe that the vector fields

$$
N_{f}=(\nabla f,-1,0), \quad N_{g}=(\nabla g, 0,-1)
$$

span $T_{\bar{F}(x)} M^{\perp}$ for every $x \in \mathbb{R}^{m}$.

If $y=\bar{F}(x)$ and $u, v \in \mathbb{R}^{m}$, then

and

$$
\left\langle h_{y}(\bar{u}, \bar{v}), N_{f}\right\rangle=\frac{\partial^{2} f}{\partial u \partial v}(x)
$$

$$
\left\langle h_{y}(\bar{u}, \bar{v}), N_{g}\right\rangle=\frac{\partial^{2} g}{\partial u \partial v}(x) .
$$


Writing $h_{y}(u, v)=a(u, v) N_{f}+b(u, v) N_{g}$, we find

where $D=\left|N_{f}\right|^{2}\left|N_{g}\right|^{2}-\left\langle N_{f}, N_{g}\right\rangle^{2}$.

$$
\begin{gathered}
a(u, v)=D^{-1}\left(\left|N_{g}\right|^{2} \frac{\partial^{2} f}{\partial u \partial v}-\left\langle N_{f}, N_{g}\right\rangle \frac{\partial^{2} g}{\partial u \partial v}\right) \\
b(u, v)=D^{-1}\left(-\left\langle N_{f}, N_{g}\right\rangle \frac{\partial^{2} f}{\partial u \partial v}+\left|N_{f}\right|^{2} \frac{\partial^{2} g}{\partial u \partial v}\right),
\end{gathered}
$$

Thus dim Image $h_{y}=1$ implies there exist smooth functions $\tilde{\alpha}, \tilde{\beta}: U \rightarrow \mathbb{R}$ on a neighborhood of $0 \in \mathbb{R}^{m},(\tilde{\alpha}, \tilde{\beta}) \neq(0,0)$, such that for all $u, v \in \mathbb{R}^{m}$,

$$
\tilde{\alpha} a(u, v)+\tilde{\beta} b(u, v)=0 .
$$

Plugging in the expressions for $a, b$ we find that there exist smooth functions $\alpha, \beta: U \rightarrow \mathbb{R},(\alpha, \beta) \neq(0,0)$ such that

$$
\alpha(x) \nabla^{2} f(x)+\beta(x) \nabla^{2} g(x)=0 .
$$

We may assume $\beta(0) \neq 0$, and so setting $\gamma=-\alpha / \beta$ we find

$$
\nabla^{2} g=\gamma \nabla^{2} f
$$

If $\gamma$ is constant near 0 , it follows that $g=\gamma f$ near 0 , so $M$ locally lies in a hyperplane. Otherwise, $0 \in \operatorname{supp}(\nabla \gamma)$, and we choose a sequence $\mathbb{R}^{m} \ni z_{k} \rightarrow 0$ with $\nabla \gamma\left(z_{k}\right) \neq 0$. Given $k$, we can find Euclidean coordinates $x_{i}$ on $\mathbb{R}^{m}$ such that $\frac{\partial^{2} f}{\partial x_{i} \partial x_{j}}\left(z_{k}\right)=0$ for all $i \neq j$. By assumption, there is $i=i(k)$ such that $\frac{\partial \gamma}{\partial x_{i}}\left(z_{k}\right) \neq 0$. From (24) we find

$$
\frac{\partial \gamma}{\partial x_{i}} \frac{\partial^{2} f}{\partial x_{j}^{2}}=\frac{\partial \gamma}{\partial x_{j}} \frac{\partial^{2} f}{\partial x_{i} \partial x_{j}}
$$

so that for all $j \neq i$ we have $\frac{\partial^{2} f}{\partial x_{j}^{2}}\left(z_{k}\right)=0$. Thefefore $\operatorname{rank} \nabla f^{2}\left(z_{k}\right) \leq 1$ for all $k$, and consequently rank $\nabla f^{2}(0) \leq 1$. This readily implies that $h_{p}$ has rank at most one, and since $h_{p} \neq 0$ we conclude $\operatorname{rank} h_{p}=1$.

We will also need a fact from linear algebra.

Lemma 5.11. Let $h: \operatorname{Sym}^{2}(V) \rightarrow W$ be a linear map. If $\operatorname{dim} \operatorname{Image} h \leq 2 \leq \operatorname{dim} V$, then there exists an open and dense subset $U \subset V$, such that for every $u \in U$, the map $V \rightarrow W, v \mapsto h(u, v)$ is onto Image $h$.

Proof. Let us first assume that $\operatorname{dim} \operatorname{Image} h=1$. The set of all $u \in V$ such that $h(u, v)=0$ for all $v \in V$ is a linear subspace of $V$. Since $h \neq 0$, this subspace must be different from $V$. Its complement is therefore open and dense.

Let us assume now that $\operatorname{dim}$ Image $h=2$. Note that without loss of generality we may assume that $h$ is surjective, that is $\operatorname{dim} W=2$. Choose a basis $w_{1}, w_{2}$ of $W$. With respect to this basis write $h=\left(h^{1}, h^{2}\right)$. For every $u \in V$ we have $h^{1}(u, \bullet), h^{2}(u, \bullet) \in V^{*}$. Let $Z$ be the set of all $u \in V$ such that the functionals $h^{1}(u, \bullet), h^{2}(u, \bullet) \in V^{*}$ are linearly dependent. Clearly $Z$ is the zero set of a system of polynomial equations on $V$. Let $U$ be its complement. Then either $U=\emptyset$, or $U$ is open and dense. In the latter case we are done. 
Otherwise, $h^{1}(u, \bullet), h^{2}(u, \bullet) \in V^{*}$ are linearly dependent for every $u \in V$. This implies that for every $u \in V$ there exists a non-zero $\lambda(u) \in W^{*}$ such that $h(u, v) \in$ $\operatorname{ker} \lambda(u)$ for every $v \in V$.

Let $v_{1}, v_{2} \in V$. If $h\left(v_{1}, v_{2}\right)=0$, then since $\left\{h\left(v_{1}+v_{2}, u\right): u \in V\right\}$ is contained in the line $\operatorname{Ker}\left(\lambda\left(v_{1}+v_{2}\right)\right)$, we have in particular that $h\left(v_{1}+v_{2}, v_{1}\right)=h\left(v_{1}, v_{1}\right)$, $h\left(v_{1}+v_{2}, v_{2}\right)=h\left(v_{2}, v_{2}\right)$ are proportional. If $h\left(v_{1}, v_{2}\right) \neq 0$, then since $h\left(v_{1}, v_{2}\right)=$ $h\left(v_{2}, v_{1}\right)$, we must have $\operatorname{ker} \lambda\left(v_{1}\right)=\operatorname{ker} \lambda\left(v_{2}\right)$, hence $h\left(v_{1}, v_{1}\right)$ and $h\left(v_{2}, v_{2}\right)$ must again be proportional. We conclude that $\operatorname{dim} \operatorname{Image} h=1$, in contradiction.

Proof of Theorem $A$. Recall that by Proposition 2.7, we only need to consider $\mu_{1}^{F}$. Let us show that all $A_{\sigma \tau}^{k}(p, u)$ are determined by the restriction of $F$ to a neighborhood of $p$ in $M$.

Assume first $n=m+1$, and consider $p \in M$. If $h_{p}=0$ then $\partial^{k} F / \partial h_{p}\left(v_{1}, v_{2}\right)^{k}=0$ for all $k \geq 1$ and $v_{1}, v_{2} \in T_{p} M$, and we conclude using Corollary 4.9. If $h_{p} \neq 0$, it holds by Lemma 5.11 that Image $h_{p}(u, \bullet)=$ Image $h_{p}=T_{p} M^{\perp}$ in a dense set of $(p, u) \in S M$ near $p$. Proposition 5.8 now concludes this case.

Assume now $n=m+2$, and fix $p \in M$. If $h_{p}=0$, we conclude as in the previous case. If $h_{p} \neq 0$, then by the lower semicontinuity of dim Image $h_{x}$ either $\operatorname{dim}$ Image $h_{x}=1$ in a neighborhood of $p$, or $p$ is a limit point of $\left\{x \in M:\right.$ Image $h_{x}=$ $T_{x} M^{\perp}$. Hence by Lemma 5.10, one of the following three must hold:

(1) $p$ is a limit point of $W:=\{x \in M$ : a neighborhood of $x$ lies in a hyperplane $\}$.

(2) For every $x \in M$ in a neighborhood of $p$, it holds that dim Image $\left(h_{x}\right)=1$, and for any $N \in T_{x} M^{\perp}$ we have $\operatorname{rank}\left\langle h_{x}, N\right\rangle \leq 1$.

(3) $p$ is a limit point of $\left\{x \in M:\right.$ Image $\left.h_{x}=T_{x} M^{\perp}\right\}$.

In the first case, we use the previously considered hypersurface case and the linear Weyl principle to conclude that for some neighborhood $U$ of $p, A_{\sigma \tau}^{k}$ is determined in $U \cap W$ by the restriction of $F$ to $U$, and by continuity it is also determined at $p$. In the second case it follows from (8) that $A_{\sigma \tau}^{k}(u)=0$ for $u \in S_{p} M$ and $k \geq 2$. Recall also that $A^{0}(u)$ is intrinsically determined by Corollary 4.4, By Lemma 5.11, it holds in a dense set of $(x, u)$ in $S M$ near $p$ that Image $h_{x}=$ Image $h_{x}(u, \bullet)$; hence by Lemma 5.9 all coefficients $A_{\sigma \tau}^{1}(u)$ are intrinsically defined, concluding the proof in this case. In the last case, we conclude with Lemma 5.11 and Proposition 5.8 .

Finally for $m+3 \leq n \leq 2 m$, the statement follows from Proposition 5.8 and Lemma 5.3 .

\section{Concluding REMARKS AND OPEn PROBlems}

One remaining open question is whether the full Weyl principle holds in codimensions one and two, namely whether the restriction of the Holmes-Thompson intrinsic volumes is independent of the isometric immersion $M \rightarrow V$. While at first glance it appears unlikely due to the appearance of the second fundamental form in (8), rigidity phenomena might have the final say in this matter.

For instance, Weyl's theorem for generic Riemannian manifolds of dimension at least three that are embedded as hypersurfaces in $\mathbb{R}^{n}$ is trivial, since in this case the first fundamental form determines the second fundamental form up to sign, see 
[36. Assuming a similar phenomenon occurs in the Finsler setting, the validity of the full Weyl principle appears plausible in light of the weak Weyl principle for hypersurfaces. In codimension two no such rigidity takes place in the Riemannian setting, and consequently we conjecture that the full Weyl principle fails in the Finslerian setting in codimension two.

In addition, it remains unknown for $m+3 \leq n \leq 2 m$ whether the set of immersions $M^{m} \rightarrow V^{n}$ for which WWP holds contains in fact a dense open set, rather than merely a residual set, as established in Theorem A.

\section{REFERENCES}

[1] S. Alesker, On P. McMullen's conjecture on translation invariant valuations, Adv. Math. 155 (2000), no. 2, 239-263. MR1794712

[2] _ Valuations on manifolds and integral geometry, Geom. Funct. Anal. 20 (2010), no. 5, 1073-1143.

[3] - Theory of valuations on manifolds. IV. New properties of the multiplicative structure, Geometric aspects of functional analysis, Lecture Notes in Math., vol. 1910, Springer, Berlin, 2007, pp. 1-44.

[4] S. Alesker and A. Bernig, The product on smooth and generalized valuations, Amer. J. Math. 134 (2012), no. 2, 507-560. MR2905004

[5] S. Alesker and J. Bernstein, Range characterization of the cosine transform on higher Grassmannians, Adv. Math. 184 (2004), no. 2, 367-379. MR2054020

[6] S. Alesker and J. H. G. Fu, Integral geometry and valuations, Advanced Courses in Mathematics. CRM Barcelona, Birkhäuser/Springer, Basel, 2014. Lectures from the Advanced Course on Integral Geometry and Valuation Theory held at the Centre de Recerca Matemàtica (CRM), Barcelona, September 6-10, 2010; Edited by Eduardo Gallego and Gil Solanes. MR3380549

[7] S. Alesker, Introduction to the theory of valuations, CBMS Regional Conference Series in Mathematics, vol. 126, Published for the Conference Board of the Mathematical Sciences, Washington, DC; by the American Mathematical Society, Providence, RI, 2018. MR3820854

[8] J. C. Álvarez Paiva, Some problems on Finsler geometry, Handbook of differential geometry. Vol. II, Elsevier/North-Holland, Amsterdam, 2006, pp. 1-33. MR2194667

[9] J. C. Álvarez Paiva and E. Fernandes, Crofton formulas in projective Finsler spaces, Electron. Res. Announc. Amer. Math. Soc. 4 (1998), 91-100. MR1655987

[10] Gelfand transforms and Crofton formulas, Selecta Math. (N.S.) 13 (2007), no. 3, 369390. MR2383600

[11] J. C. Álvarez Paiva and A. C. Thompson, Volumes on normed and Finsler spaces, A sampler of Riemann-Finsler geometry, Math. Sci. Res. Inst. Publ., vol. 50, Cambridge Univ. Press, Cambridge, 2004, pp. 1-48. MR2132656

[12] A. Bernig, Valuations with Crofton formula and Finsler geometry, Adv. Math. 210 (2007), no. 2, 733-753. MR2303237

[13] A. Bernig and L. Bröcker, Valuations on manifolds and Rumin cohomology, J. Differential Geom. 75 (2007), no. 3, 433-457. MR2301452

[14] A. Bernig, D. Faifman, and G. Solanes, Curvature Measures of Pseudo-Riemannian manifolds, preprint.

[15] A. Bernig, J. H. G. Fu, and G. Solanes, Integral geometry of complex space forms, Geom. Funct. Anal. 24 (2014), no. 2, 403-492, DOI 10.1007/s00039-014-0251-1. MR3192033

[16] D. Burago and S. Ivanov, Isometric embeddings of Finsler manifolds, Algebra i Analiz 5 (1993), no. 1, 179-192.

[17] _ On intrinsic geometry of surfaces in normed spaces, Geom. Topol. 15 (2011), no. 4, 2275-2298. MR2862157

[18] D. Faifman, Contact integral geometry and the Heisenberg algebra. 
[19] J. H. G. Fu, Curvature measures of subanalytic sets, Amer. J. Math. 116 (1994), no. 4, 819-880. MR1287941

[20] - Integral geometric regularity, Tensor valuations and their applications in stochastic geometry and imaging, Lecture Notes in Math., vol. 2177, Springer, Cham, 2017, pp. 261-299. MR3702376

[21] J. H. G. Fu, D. Pokorný, and J. Rataj, Kinematic formulas for sets defined by differences of convex functions, Adv. Math. 311 (2017), 796-832. MR3628231

[22] P. Garret, Harmonic analysis on spheres, II, available at http://www-users.math.umn.edu/ garrett/m/mfms/notes_c/spheres_II.pdf

[23] M. Golubitsky and V. Guillemin, Stable mappings and their singularities, Springer-Verlag, New York-Heidelberg, 1973. Graduate Texts in Mathematics, Vol. 14. MR0341518

[24] H. Groemer, Geometric applications of Fourier series and spherical harmonics, Encyclopedia of Mathematics and its Applications, vol. 61, Cambridge University Press, Cambridge, 1996.

[25] C.-H. Gu, Imbedding of a Finsler space in a Minkowski space, Acta Math. Sinica 6 (1956), 215-232 (Chinese, with Russian summary). MR100889

[26] L. Hörmander, The analysis of linear partial differential operators. I, Classics in Mathematics, Springer-Verlag, Berlin, 2003. Distribution theory and Fourier analysis; Reprint of the second (1990) edition [Springer, Berlin; MR1065993 (91m:35001a)]. MR1996773

[27] S. Ivanov, Monochromatic Finsler surfaces and a local ellipsoid characterization, Proc. Amer. Math. Soc. 146 (2018), no. 4, 1741-1755.

[28] D. A. Klain, A short proof of Hadwiger's characterization theorem, Mathematika 42 (1995), no. 2, 329-339. MR1376731

[29] M. Ludwig, Minkowski areas and valuations, J. Differential Geom. 86 (2010), no. 1, 133-161. MR2772547

[30] B. Rubin, Inversion and characterization of the hemispherical transform, J. Anal. Math. 77 (1999), 105-128.

[31] R. Schneider, Convex bodies: the Brunn-Minkowski theory, Second expanded edition, Encyclopedia of Mathematics and its Applications, vol. 151, Cambridge University Press, Cambridge, 2014. MR3155183

[32] R. Schneider and J. A. Wieacker, Integral geometry in Minkowski spaces, Adv. Math. 129 (1997), no. 2, 222-260. MR1462734

[33] Z. Shen, On Finsler geometry of submanifolds, Math. Ann. 311 (1998), no. 3, 549-576. MR1637939

[34] G. Solanes and T. Wannerer, Integral geometry of exceptional spheres, J. Differential Geom., in press, available at arXiv:1708.05861 [math.DG].

[35] M. Spivak, A comprehensive introduction to differential geometry. Vol. IV, 2nd ed., Publish or Perish, Inc., Wilmington, Del., 1979. MR532833

[36] _ A comprehensive introduction to differential geometry. Vol. V, 2nd ed., Publish or Perish, Inc., Wilmington, Del., 1979. MR532834

[37] A. C. Thompson, Minkowski geometry, Encyclopedia of Mathematics and its Applications, vol. 63, Cambridge University Press, Cambridge, 1996. MR1406315

[38] H. Weyl, On the Volume of Tubes, Amer. J. Math. 61 (1939), no. 2, 461-472.

E-mail address: dmitry.faifman@umontreal.ca

E-mail address: thomas.wannerer@uni-jena.de

FAKUltät Für Mathematik und Informatik, Friedrich-Schiller-Universität Jena, 07743 Jena, Germany

Centre de recherches mathématiques, Université de Montréal, Pavillon AndréAisenstadt, 2920, Chemin de la tour, Bur. 5357 Montréal (Québec) H3T 1J4 Canada 